\title{
Epidemics in Two Competing Species
}

\author{
Litao $\mathrm{Han}^{1}$
}

School of Information, Renmin University of China, Beijing, 100872 P. R. China

\author{
Andrea Pugliese ${ }^{2}$ \\ Department of Mathematics, University of Trento, Trento, 38050 Italy
}

\begin{abstract}
An SIS epidemic model in two competing species with the mass action incidence is formulated and analyzed. Thresholds for the existence of boundary equilibria are identified and conditions for their local asymptotic stability or instability are found. By persistence theory, conditions for the persistence of either hosts or pathogens are proved. Using Hopf bifurcation theory and numerical simulations, some aspects of the complicated dynamical behaviors of the model are shown: the system may have zero up to three internal equilibria, may have a stable limit cycle, may have three stable attractors. Through the results on persistence and stability of the boundary equilibria, some important interactions between infection and competition are revealed: (1) a species that would get extinct without the infection, may persist in presence of the infection; (2) a species that would coexist with its competitor without the infection, is driven to extinction by the infection; (3) an infection that would die out in either species without the inter-infection of disease, may persist in both species in presence of this factor.
\end{abstract}

Key words: Epidemic model, Multi-host infection, Species extinction, Uniform persistence, Hopf bifurcation, Periodic solutions 


\section{Introduction}

Models for ecological interactions, and models for host-pathogen interactions were initially developed separately. However, it has been recognized that a strong interaction may arise between these factors: a pathogen may tilt the balance between competing species, or may provoke a negative influence between the densities of two species that are not otherwise interacting ("apparent competition"), even causing the extinction of one of them; a pathogen may be able to persist in a community of 2 (or more) competing species, but not in any of them in isolation.

The problem, however, is that the models become quickly very difficult to analyse. Even the simplest possible model with an SI epidemics spreading in two not interacting species [1] gives rise to a 4-dimensional ODE model that may have multiple stable attractors often in the form of limit cycles [2].

It must be however remarked that the behavior of the model becomes much simpler [3] if one assumes that infection incidence is proportional to the infected fractions in each species and not to the densities of infectives. Which is the most appropriate form for the infection rate has been debated in sev-

\footnotetext{
Email addresses: hanlitao@ruc.edu.cn (Litao Han), pugliese@science.unitn.it (Andrea Pugliese).

1 Partially supported by the National Natural Sciences Foundation of China (No. 10501052). This manuscript was prepared while L. H. was visiting the University of Trento with the partial support of the Italian Foreign Ministry

2 Supported in part by the project EPICO of the Province of Trento, Italy
} 
eral papers, and the conclusion should clearly depend on the actual transmission routes on the pathogen. Considering a generic infection that is indirectly (through air-borne propagules or through the soil) transmitted among competing species, we believe that the standard bilinear form (mass action incidence) used in [1] is quite reasonable, and we will use it in our model.

Some empirical investigations on epidemics in competing species have been performed, with the help of epidemic models, by Begon et al. [4] on the cowpox virus in coexisting populations of bank voles and wood mice, and by Tompkins et al. [5] on a pathogen in competing squirrel species. Begon et al. [6] suggest that the data on the presence of the pathogen in islands are not compatible with the mass action incidence, but we believe that several different interpretation of these data are possible.

Models for two species which share a disease without competition have been discussed in some papers. In their works, Holt and Pickering [7], Begon and Bowers $[1,8]$ conjectured that the models have the classic endemic model behavior: the infected coexistence equilibrium is relevant and stable if and only if no other stable equilibrium exists in the nonnegative orthant. But this conjecture was soon denied by some counterexamples given by Greenman and Hudson [2]. On the other hand, Hethcote et al. [9] later found that the models have the behavior of a classic endemic model if the frequency-dependent incidence is used.

Epidemic models in competing species have also been studied previously. Anderson and May [10] considered a host-competitor-pathogen model which involves two direct competitors, one subject to a pathogen. They examined the effect of a pathogen on conventional competition. Bowers and Turner [11] 
introduced a proto-typical model of two hosts sharing a pathogen and competing directly. They studied the interplay between infection and competition. In their model, the death rates are density-independent. Venturino [12] analyzed the dynamics of two competing species when one of them is subject to a disease. In his model with mass action incidence, he obtained limit cycles. Han et al. [13] studied an SIRS epidemic model of two competitive species without disease-related deaths. They analyzed the effect of inter-infection of disease on the dynamical behaviors of the model. Saenz and Hethcote [3] considered some models of SIS, SIR and SIRS type with frequency-dependent incidence. They found that the models have the classic endemic model behavior. In their paper, a key result is that the disease must either die out in both species or remain endemic in both species.

Our model is different from the previous models, because it uses the mass action incidence, both density-dependent and disease-related death rates, and both species can be infected. Because of the complicated behaviors of the system, shown by [2], we do not aim at a complete analysis. Rather, instead of considering the global stabilities of the equilibria, we focus on the concept of persistence, and present conditions that guarantee the persistence (or the non-persistence) of either hosts or pathogens, following ideas by Thieme [14]. Together with the discussion on the local stabilities of the equilibria, by the analysis of persistence, we investigate the interactions between infection and competition. Moreover, by Hopf bifurcation theory and numerical simulations, some complex behaviors of the model are shown.

The organization of this paper is as follows. in the next section, we introduce the model and some preliminary results. In section 3, the existences and stabilities of the equilibria are discussed. In section 4, we analyze the persistence 
of either hosts or pathogens. In section 5, we study the bifurcation phenomena and analyze the periodic orbits and the number of the internal equilibria. Finally, in section 6, the detailed discussion and conclusions are given.

\section{Formulation of the Model}

We consider two competing species, whose densities are denoted by $N_{1}$ and $N_{2}$, whose dynamics follow a standard Lotka-Volterra system. $K_{i}$ are the carrying capacity of each species in isolation, $r_{i}=b_{i}-d_{i}$ the intrinsic growth rates, $\alpha_{12}$ and $\alpha_{21}$ the competition coefficients. When considering the spread of an infectious disease in a species that grows logistically, it becomes necessary to specify explicitly the birth and the death rate; we follow Gao and Hethcote [15], assuming that $b_{i}-\frac{a_{i} r_{i} N_{i}}{K_{i}}$ is the birth rate and $\left[d_{i}+\left(1-a_{i}\right) \frac{r_{i} N_{i}}{K_{i}}\right]$ the death rate of species $i$ in isolation, where $0 \leq a_{i} \leq 1$ is a parameter that subdivides the density-dependence between births and deaths (note that for $a_{i}>0$ the birth rates become negative at very high densities, which may be disturbing; however, such densities are outside the compact attracting set in which the analysis is relevant). Competition will be assumed to act only on death rates, so that the death rate of species $i$ when its own density is $N_{i}$ and that of the competitor is $N_{j}$ are $d_{i}+\left(1-a_{i}\right) \frac{r_{i} N_{i}}{K_{i}}+\alpha_{i j} \frac{r_{i} N_{j}}{K_{i}}$.

We now assume that both species can be infected by a common pathogen, whose cycle follow an SIS scheme, i.e. following recovery an individual become susceptible and can be infected again. Each species will then be divided in a susceptible part $S_{i}$ and an infected class $Y_{i}$. We let $\beta_{i i}$ be the intrainfection rate of disease in species $i$, and $\beta_{i j}(i \neq j)$ the inter-infection rate of disease between the two species, both following the mass action incidence 
rule. Moreover, $\gamma_{i}$ is the recovery rate and $\delta_{i}$ is the disease-related death rate.

We assume $\delta_{i}>0$ (otherwise, the system's behavior would be much simpler), but neglect any effect of the infection on the birth rates.

From these assumptions, we obtain the following system:

$$
\left\{\begin{aligned}
\frac{d S_{1}}{d t}= & \left(b_{1}-\frac{a_{1} r_{1} N_{1}}{K_{1}}\right) N_{1}-\left[d_{1}+r_{1}\left(\frac{\left(1-a_{1}\right) N_{1}+\alpha_{12} N_{2}}{K_{1}}\right)\right] S_{1} \\
& -S_{1}\left(\beta_{11} Y_{1}+\beta_{12} Y_{2}\right)+\gamma_{1} Y_{1} \\
\frac{d Y_{1}}{d t}= & S_{1}\left(\beta_{11} Y_{1}+\beta_{12} Y_{2}\right)-\gamma_{1} Y_{1}-\left[d_{1}+r_{1}\left(\frac{\left(1-a_{1}\right) N_{1}+\alpha_{12} N_{2}}{K_{1}}+\delta_{1}\right)\right] Y_{1} \\
\frac{d N_{1}}{d t}= & r_{1}\left(1-\frac{N_{1}}{K_{1}}-\frac{\alpha_{12} N_{2}}{K_{1}}\right) N_{1}-\delta_{1} Y_{1} \\
& -S_{2}\left(\beta_{21} Y_{1}+\beta_{22} Y_{2}\right)+\gamma_{2} Y_{2} \\
\frac{d S_{2}}{d t}= & \left(b_{2}-\frac{a_{2} r_{2} N_{2}}{K_{2}}\right) N_{2}-\left[d_{2}+r_{2}\left(\frac{\left(1-a_{2}\right) N_{2}+\alpha_{21} N_{1}}{K_{2}}\right)\right] S_{2} \\
\frac{d Y_{2}}{d t}= & S_{2}\left(\beta_{21} Y_{1}+\beta_{22} Y_{2}\right)-\gamma_{2} Y_{2}-\left[d_{2}+r_{2}\left(\frac{\left(1-a_{2}\right) N_{2}+\alpha_{21} N_{1}}{K_{2}}+\delta_{2}\right)\right] Y_{2} \\
\frac{d N_{2}}{d t}= & r_{2}\left(1-\frac{N_{2}}{K_{2}}-\frac{\alpha_{21} N_{1}}{K_{2}}\right) N_{2}-\delta_{2} Y_{2}
\end{aligned}\right.
$$

System (2.1) comprises 6 equations, but only 4 are necessary, since $N_{i}=S_{i}+Y_{i}$.

We choose to use as variables $N_{i}$ and $I_{i}=\frac{Y_{i}}{N_{i}}$ for $i=1,2$, obtaining the 
following 4 dimensional system:

$$
\left\{\begin{aligned}
\frac{d I_{1}}{d t}= & {\left[\beta_{11}\left(1-I_{1}\right) N_{1}-\left(b_{1}-\frac{a_{1} r_{1} N_{1}}{K_{1}}\right)-\left(\gamma_{1}+\delta_{1}\right)+\delta_{1} I_{1}\right] I_{1} } \\
& +\beta_{12}\left(1-I_{1}\right) I_{2} N_{2} \\
\frac{d N_{1}}{d t}= & {\left[r_{1}\left(1-\frac{N_{1}}{K_{1}}-\frac{\alpha_{12} N_{2}}{K_{1}}\right)-\delta_{1} I_{1}\right] N_{1} } \\
\frac{d I_{2}}{d t}= & {\left[\beta_{22}\left(1-I_{2}\right) N_{2}-\left(b_{2}-\frac{a_{2} r_{2} N_{2}}{K_{2}}\right)-\left(\gamma_{2}+\delta_{2}\right)+\delta_{2} I_{2}\right] I_{2} } \\
& +\beta_{21}\left(1-I_{2}\right) I_{1} N_{1} \\
\frac{d N_{2}}{d t}= & {\left[r_{2}\left(1-\frac{N_{2}}{K_{2}}-\frac{\alpha_{21} N_{1}}{K_{2}}\right)-\delta_{2} I_{2}\right] N_{2} } \\
0 \leq & I_{i} \leq 1, \quad 0 \leq N_{i} \quad i=1,2
\end{aligned}\right.
$$

It is easy to see that system (2.2) is mathematically well posed in the positive invariant region $D=\left\{\left(I_{1}, N_{1}, I_{2}, N_{2}\right) \mid 0 \leq I_{i} \leq 1,0 \leq N_{i} \leq K_{i}, i=1,2\right\}$ and solutions in $D$ exist for all positive time. In the remainder of the paper, system (2.2) is always analyzed in the region $D$.

Before proceeding, we briefly summarize known results on the two ingredients of system (2.2), the Lotka-Volterra competition model, and the host-pathogen model.

\subsection{Basic results on Lotka-Volterra competition model}

Consider first the classic Lotka-Volterra competition model

$$
\left\{\begin{array}{l}
\frac{d N_{1}}{d t}=r_{1}\left(1-\frac{N_{1}}{K_{1}}-\frac{\alpha_{12} N_{2}}{K_{1}}\right) N_{1} \\
\frac{d N_{2}}{d t}=r_{2}\left(1-\frac{N_{2}}{K_{2}}-\frac{\alpha_{21} N_{1}}{K_{2}}\right) N_{2}
\end{array}\right.
$$


The following result can be found in all books on theoretical ecology.

Lemma 2.1 System (2.3) always has the equilibria $E_{0}=(0,0), E_{1}=\left(K_{1}, 0\right)$ and $E_{2}=\left(0, K_{2}\right)$. Assuming that all parameters are positive, $E_{0}$ is always unstable. As for the existence of an internal equilibrium and the stability of them all, there are 4 generic cases:

(1) If $\alpha_{12} K_{2}<K_{1}$ and $\alpha_{21} K_{1}<K_{2}$, there exists also a unique internal equilibrium $E_{12}=\left(N_{1 E}, N_{2 E}\right) . E_{12}$ is globally asymptotically stable in $\Omega=\left\{\left(N_{1}, N_{2}\right) \mid 0<N_{i} \leq K_{i}, i=1,2\right\}$.

(2) If $\alpha_{12} K_{2}>K_{1}$ and $\alpha_{21} K_{1}>K_{2}$, there exists a unique internal equilibrium $E_{12}$, which is a saddle point. Both $E_{1}$ and $E_{2}$ are locally asymptotically stable.

(3) If $\alpha_{12} K_{2}<K_{1}$ and $\alpha_{21} K_{1}>K_{2}$, there is no internal equilibrium, and $E_{1}$ is globally asymptotically stable in $\Omega$.

(4) If $\alpha_{12} K_{2}>K_{1}$ and $\alpha_{21} K_{1}<K_{2}$, there is no internal equilibrium, and $E_{2}$ is globally asymptotically stable in $\Omega$.

The coordinates of $E_{12}$ are:

$$
N_{1 E}=\frac{K_{1}-\alpha_{12} K_{2}}{1-\alpha_{12} \alpha_{21}}, \quad N_{2 E}=\frac{K_{2}-\alpha_{21} K_{1}}{1-\alpha_{12} \alpha_{21}}
$$

\subsection{SIS host-pathogen system}

Restricting system (2.2) to a single host species, one obtains the following SIS model, already considered in [16]

$$
\left\{\begin{aligned}
\frac{d I}{d t} & =\left[\beta(1-I) N-\left(b-\frac{a r N}{K}\right)-(\gamma+\delta)+\delta I\right] I \\
\frac{d N}{d t} & =\left[r\left(1-\frac{N}{K}\right)-\delta I\right] N
\end{aligned}\right.
$$


The state space for the variables is $0 \leq I \leq 1, \quad 0 \leq N$, and we assume $r=b-d>0,0 \leq a \leq 1$.

The behavior of system (2.4) can be obtained on the basis of the reproduction number

$$
R=\frac{\beta K}{b+\gamma+\delta-a r}
$$

of the infection [17]; note that the denominator can be written as $d+r(1-a)+$ $\gamma+\delta$; hence, it is positive, and represents the average length of the infectious period.

Precisely, one has

Lemma $2.2([\mathbf{1 6 , 1 8}])$ For system (2.4), if $R \leq 1$, the disease-free equilibrium $E_{1}=(0, K)$ is globally asymptotically stable in the region $\{(I, N) \mid 0 \leq$ $I \leq 1,0<N\}$.

If $R>1$, there exists a unique internal equilibrium $P=\left(I^{*}, N^{*}\right)$ which is globally asymptotically stable in the region $\{(I, N) \mid 0<I \leq 1,0<N\}$. Any solution starting from the $I$ axis goes to $E_{0}=(0,0)$, while those starting from the $N$ axis go to $E_{1}$.

Remark 2.3 Setting the RHS of (2.4) equal to 0, one sees that $\left(I^{*}, N^{*}\right)$ can be found as follows:

$$
N^{*}=K\left(1-\frac{\delta}{r} I^{*}\right) \quad F\left(I^{*}\right)=0
$$

where

$$
F(x)=\frac{\delta}{r} x^{2}-A x+\frac{R-1}{R} \text { with } A=1+\frac{\delta}{r}-\frac{\delta(1-a)}{\beta K}
$$


It is immediate to see that, since $R>1, F(0)>0$, while

$$
F(1)=\frac{\delta(1-a)}{\beta K}-\frac{1}{R}=-\frac{a \delta+b+\gamma-a r}{\beta K}=-\frac{a \delta+d+\gamma+r(1-a)}{\beta K}<0 .
$$

Hence from (2.6) one finds a unique $I^{*}$ in the feasible interval $(0,1)$, that can be written as

$$
I^{*}=\frac{r}{\delta} \frac{A-\sqrt{A^{2}-4 \frac{\delta}{r} \frac{R-1}{R}}}{2} .
$$

Moreover one can see that $\frac{\delta}{r} I^{*}<1$ since

$$
F\left(\frac{r}{\delta}\right)=\frac{r(1-a)}{\beta K}-\frac{1}{R}=-\frac{\delta+d+\gamma}{\beta K}<0 .
$$

Hence, expression (2.6) shows that $N^{*}>0$.

\section{$3 \quad$ Equilibria}

\subsection{Existence of the equilibria}

System (2.2) always has the following 3 boundary equilibria:

$$
E_{0}=(0,0,0,0), \quad E_{1}=\left(0, K_{1}, 0,0\right), \quad E_{2}=\left(0,0,0, K_{2}\right) .
$$

Let now

$$
R_{1}=\frac{\beta_{11} K_{1}}{b_{1}+\gamma_{1}+\delta_{1}-a_{1} r_{1}}, \quad R_{2}=\frac{\beta_{22} K_{2}}{b_{2}+\gamma_{2}+\delta_{2}-a_{2} r_{2}}
$$

be the reproductive ratios of the infection, analogously to (2.5).

If $R_{1}>1$ [or $R_{2}>1$ ], the following boundary equilibrium exists:

$$
B_{1}=\left(I_{1}^{*}, N_{1}^{*}, J_{2}^{*}, 0\right) \quad\left[\text { or } B_{2}=\left(J_{1}^{*}, 0, I_{2}^{*}, N_{2}^{*}\right)\right] .
$$


With the same procedure of Remark 2.3, one sees that $I_{i}^{*}$ and $N_{i}^{*}$ satisfy

$$
N_{i}^{*}=K_{i}\left(1-\frac{\delta_{i}}{r_{i}} I_{i}^{*}\right) \quad F_{i}\left(I_{i}^{*}\right)=0
$$

where

$$
F_{i}(x)=\frac{\delta_{i}}{r_{i}} x^{2}-A_{i} x+\frac{R_{i}-1}{R_{i}} \quad \text { with } A_{i}=1+\frac{\delta_{i}}{r_{i}}-\frac{\delta_{i}\left(1-a_{i}\right)}{\beta_{i i} K_{i}}
$$

Finally, $J_{i}^{*}$ can be found as the smaller solution of $G_{i}(x)=0$, where

$$
G_{i}(x)=\delta_{i} x^{2}-\left(b_{i}+\gamma_{i}+\delta_{i}+\beta_{i j} I_{j}^{*} N_{j}^{*}\right) x+\beta_{i j} I_{j}^{*} N_{j}^{*}
$$

and $j$ indicates the index different from $i$. It is in fact immediate to see that $G_{i}(x)=0$ has a unique solution in $(0,1)$ given by

$$
J_{i}^{*}=\frac{b_{i}+\gamma_{i}+\delta_{i}+\beta_{i j} I_{j}^{*} N_{j}^{*}-\sqrt{\left(b_{i}+\gamma_{i}+\delta_{i}+\beta_{i j} I_{j}^{*} N_{j}^{*}\right)^{2}-4 \delta_{i} \beta_{i j} I_{j}^{*} N_{j}^{*}}}{2 \delta_{i}} .
$$

From Section 2.1 we see that there may exist an infection-free coexistence equilibrium $E_{12}$ :

$$
E_{12}=\left(0, N_{1 E}, 0, N_{2 E}\right)
$$

under two different conditions:
a) $\alpha_{12} K_{2}<K_{1}$ and $\alpha_{21} K_{1}<K_{2}$;
b) $\alpha_{12} K_{2}>K_{1}$ and $\alpha_{21} K_{1}>K_{2}$.

In case a), the equilibrium is stable relatively to infection-free perturbations; in case b), it is unstable and the equilibria $E_{1}$ and $E_{2}$ are stable relatively to infection-free perturbations.

There may exist an internal equilibrium $P^{*}$. Its coordinates must satisfy the equations:

$$
I_{1}=\frac{r_{1}}{\delta_{1}}\left(1-\frac{N_{1}}{K_{1}}-\frac{\alpha_{12} N_{2}}{K_{1}}\right), \quad I_{2}=\frac{r_{2}}{\delta_{2}}\left(1-\frac{\alpha_{21} N_{1}}{K_{2}}-\frac{N_{2}}{K_{2}}\right)
$$


while $N_{1}$ and $N_{2}$ can be found as solution of a system of 2 algebraic equations.

As shown in $[1,2]$ (in the case without competition), there are not simple conditions that guarantee the existence of an internal equilibrium. Moreover, there exist cases in which there are several internal equilibria [2]. Hence, we do not attempt to characterize conditions for their existence, and we will limit ourselves to show some computer-assisted bifurcation diagrams, and to give sufficient conditions for their existence, based on persistence theory.

\subsection{Local stabilities of the equilibria}

In the following, we study the local stabilities of boundary equilibria. The Jacobian is

$$
A=\left[\begin{array}{cccc}
a_{11} & \beta_{11} I_{1}\left(1-I_{1}\right)+\frac{a_{1} r_{1}}{K_{1}} I_{1} & \beta_{12}\left(1-I_{1}\right) N_{2} & \beta_{12}\left(1-I_{1}\right) I_{2} \\
-\delta_{1} N_{1} & r_{1}\left(1-\frac{2 N_{1}+\alpha_{12} N_{2}}{K_{1}}\right)-\delta_{1} I_{1} & 0 & -\frac{r_{1} \alpha_{12}}{K_{1}} N_{1} \\
\beta_{21}\left(1-I_{2}\right) N_{1} & \beta_{21}\left(1-I_{2}\right) I_{1} & a_{33} & \beta_{22}\left(1-I_{2}\right) I_{2}+\frac{a_{2} r_{2}}{K_{2}} I_{2} \\
0 & -\frac{r_{2} \alpha_{21}}{K_{2}} N_{2} & -\delta_{2} N_{2} & r_{2}\left(1-\frac{\alpha_{21} N_{1}+2 N_{2}}{K_{2}}\right)-\delta_{2} I_{2}
\end{array}\right]
$$

where

$$
\begin{aligned}
& a_{11}=\beta_{11} N_{1}-2 \beta_{11} I_{1} N_{1}-\left(b_{1}+\gamma_{1}+\delta_{1}\right)+\frac{a_{1} r_{1}}{K_{1}} N_{1}+2 \delta_{1} I_{1}-\beta_{12} I_{2} N_{2}, \\
& a_{33}=\beta_{22} N_{2}-2 \beta_{22} I_{2} N_{2}-\left(b_{2}+\gamma_{2}+\delta_{2}\right)+\frac{a_{2} r_{2}}{K_{2}} N_{2}+2 \delta_{2} I_{2}-\beta_{21} I_{1} N_{1} .
\end{aligned}
$$

For $E_{0}=(0,0,0,0)$, we have

$$
A_{0}=\left[\begin{array}{cccc}
-\left(b_{1}+\gamma_{1}+\delta_{1}\right) & 0 & 0 & 0 \\
0 & r_{1} & 0 & 0 \\
0 & 0-\left(b_{2}+\gamma_{2}+\delta_{2}\right) & 0 \\
0 & 0 & 0 & r_{2}
\end{array}\right]
$$


So $E_{0}$ is unstable.

For $E_{1}=\left(0, K_{1}, 0,0\right)$, we have

$$
A_{1}=\left[\begin{array}{cccc}
\beta_{11} K_{1}-\left(b_{1}+\gamma_{1}+\delta_{1}\right)+a_{1} r_{1} & 0 & 0 & 0 \\
-\delta_{1} K_{1} & -r_{1} & 0 & -r_{1} \alpha_{12} \\
\beta_{21} K_{1} & 0 & -\left(b_{2}+\gamma_{2}+\delta_{2}\right) & 0 \\
0 & 0 & 0 & r_{2}\left(1-\frac{\alpha_{21} K_{1}}{K_{2}}\right)
\end{array}\right]
$$

Its eigenvalues are

$\lambda_{1}=\beta_{11} K_{1}-\left(b_{1}+\gamma_{1}+\delta_{1}\right)+a_{1} r_{1}, \lambda_{2}=-r_{1}, \lambda_{3}=-\left(b_{2}+\gamma_{2}+\delta_{2}\right), \lambda_{4}=$ $r_{2}\left(1-\frac{\alpha_{21} K_{1}}{K_{2}}\right)$.

So $E_{1}$ is stable if $\alpha_{21} K_{1}>K_{2}$ and $R_{1}<1$; it is unstable if either inequality is reversed.

Analogously, one finds that $E_{2}=\left(0,0,0, K_{2}\right)$ is stable if $\alpha_{12} K_{2}>K_{1}$ and $R_{2}<1$; it is unstable if either inequality is reversed.

For $B_{2}=\left(J_{1}^{*}, 0, I_{2}^{*}, N_{2}^{*}\right)$, we have

$$
A_{B_{2}}=\left[\begin{array}{cccc}
b_{11} & \beta_{11} J_{1}^{*}\left(1-J_{1}^{*}\right)+\frac{a_{1} r_{1}}{K_{1}} J_{1}^{*} & \beta_{12}\left(1-J_{1}^{*}\right) N_{2}^{*} & \beta_{12}\left(1-J_{1}^{*}\right) I_{2}^{*} \\
0 & r_{1}\left(1-\alpha_{12} \frac{N_{2}^{*}}{K_{1}}\right)-\delta_{1} J_{1}^{*} & 0 & 0 \\
0 & \beta_{21}\left(1-I_{2}^{*}\right) J_{1}^{*} & -\left(\beta_{22} N_{2}^{*}-\delta_{2}\right) I_{2}^{*} & \beta_{22}\left(1-I_{2}^{*}\right) I_{2}^{*}+\frac{a_{2} r_{2}}{K_{2}} I_{2}^{*} \\
0 & -\alpha_{21} \frac{r_{2}}{K_{2}} N_{2}^{*} & -\delta_{2} N_{2}^{*} & r_{2}-2 \frac{r_{2}}{K_{2}} N_{2}^{*}-\delta_{2} I_{2}^{*}
\end{array}\right]
$$

where

$$
b_{11}=-\left(b_{1}+\gamma_{1}+\delta_{1}\right)+2 \delta_{1} J_{1}^{*}-\beta_{12} I_{2}^{*} N_{2}^{*} .
$$

It is immediate to see that the eigenvalues of $A_{B_{2}}$ are $\lambda_{1}=b_{11}, \lambda_{2}=r_{1}\left(1-\alpha_{12} \frac{N_{2}^{*}}{K_{1}}\right)-\delta_{1} J_{1}^{*}$, while $\lambda_{3}$ and $\lambda_{4}$ the two eigenvalues of the bottom-right submatrix

$$
B R=\left[\begin{array}{cc}
-\left(\beta_{22} N_{2}^{*}-\delta_{2}\right) I_{2}^{*} & \beta_{22}\left(1-I_{2}^{*}\right) I_{2}^{*}+\frac{a_{2} r_{2}}{K_{2}} I_{2}^{*} \\
-\delta_{2} N_{2}^{*} & r_{2}-2 \frac{r_{2}}{K_{2}} N_{2}^{*}-\delta_{2} I_{2}^{*}
\end{array}\right]
$$

Both eigenvalues of $B R$ have negative real parts, as known from the analysis 
of SIS models in a single population (see Section 2.2) and confirmed by the sign of its trace and determinant.

Moreover, we have

$$
\lambda_{1}=b_{11}=\frac{1}{J_{1}^{*}}\left(\delta_{1} J_{1}^{* 2}-\beta_{12} I_{2}^{*} N_{2}^{*}\right)<0
$$

using the fact that $J_{1}^{*}$ is the smaller root of (3.2).

Hence $B_{2}$ is stable if $\pi_{1}=r_{1}\left(1-\alpha_{12} \frac{N_{2}^{*}}{K_{1}}\right)-\delta_{1} J_{1}^{*}<0$, and unstable if the inequality is reversed.

Analogously, $B_{1}$ is stable if $\pi_{2}=r_{2}\left(1-\alpha_{21} \frac{N_{1}^{*}}{K_{2}}\right)-\delta_{2} J_{2}^{*}<0$, unstable otherwise.

For studying the eigenvalues of the Jacobian at $E_{12}$, it is more convenient to change the order of coordinates to $\left(I_{1}, I_{2}, N_{1}, N_{2}\right)$; hence, the equilibrium will be $\left(0,0, N_{1 E}, N_{2 E}\right)$. Then, its Jacobian is

$$
C_{12}=\left[\begin{array}{cccc}
c_{11} & \beta_{12} N_{2 E} & 0 & 0 \\
\beta_{21} N_{1 E} & c_{22} & 0 & 0 \\
-\delta_{1} N_{1 E} & 0 & r_{1}\left(1-\frac{2 N_{1 E}+\alpha_{12} N_{2 E}}{K_{1}}\right) & -\frac{r_{1} \alpha_{12}}{K_{1}} N_{1 E} \\
0 & -\delta_{2} N_{2 E} & -\frac{r_{2} \alpha_{21}}{K_{2}} N_{2 E} & r_{2}\left(1-\frac{\alpha_{21} N_{1 E}+2 N_{2 E}}{K_{2}}\right)
\end{array}\right]
$$

with

$c_{11}=\beta_{11} N_{1 E}-\left(b_{1}+\gamma_{1}+\delta_{1}\right)+\frac{a_{1} r_{1}}{K_{1}} N_{1 E}, \quad c_{22}=\beta_{22} N_{2 E}-\left(b_{2}+\gamma_{2}+\delta_{2}\right)+\frac{a_{2} r_{2}}{K_{2}} N_{2 E}$

Since $C_{12}$ is block triangular, its eigenvalues are those of the top-left and of the bottom-right submatrices. The top-left matrix $T L$ can be written as

$$
\begin{aligned}
& T L=K-D \quad \text { with } \\
& K=\left[\begin{array}{ll}
\beta_{11} N_{1 E} & \beta_{12} N_{2 E} \\
\beta_{21} N_{1 E} & \beta_{22} N_{2 E}
\end{array}\right] \text { and } D=\operatorname{diag}\left[\begin{array}{c}
d_{1}+\gamma_{1}+\delta_{1}+r_{1}\left(1-a_{1} \frac{N_{1 E}}{K_{1}}\right) \\
d_{2}+\gamma_{2}+\delta_{2}+r_{2}\left(1-a_{2} \frac{N_{2 E}}{K_{2}}\right)
\end{array}\right] \text {. }
\end{aligned}
$$


In conclusion, the eigenvalues of $T L$ have all negative real parts if and only if all eigenvalues of $K D^{-1}$ are in the interior of the unit ball $[19,20]$. One can then define $R_{12}$ as the largest eigenvalue of $K D^{-1}$, in formula

$$
R_{12}=\rho\left(\left[\begin{array}{l}
\frac{\beta_{11} N_{1 E}}{d_{1}+\gamma_{1}+\delta_{1}+r_{1}\left(1-a_{1} \frac{N_{1 E}}{K_{1}}\right)} \frac{\beta_{12} N_{2 E}}{d_{2}+\gamma_{2}+\delta_{2}+r_{2}\left(1-a_{2} \frac{N_{2 E}}{K_{2}}\right)} \\
\frac{\beta_{21} N_{1 E}}{d_{1}+\gamma_{1}+\delta_{1}+r_{1}\left(1-a_{1} \frac{N_{1 E}}{K_{1}}\right)} \frac{\beta_{22} N_{2 E}}{d_{2}+\gamma_{2}+\delta_{2}+r_{2}\left(1-a_{2} \frac{N_{2 E}}{K_{2}}\right)}
\end{array}\right]\right) .
$$

The bottom-right submatrix

$$
\left[\begin{array}{cc}
r_{1}\left(1-\frac{2 N_{1 E}+\alpha_{12} N_{2 E}}{K_{1}}\right) & -\frac{r_{1} \alpha_{12}}{K_{1}} N_{1 E} \\
-\frac{r_{2} \alpha_{21}}{K_{2}} N_{2 E} & r_{2}\left(1-\frac{\alpha_{21} N_{1 E}+2 N_{2 E}}{K_{2}}\right)
\end{array}\right]
$$

is the Jacobian of the infection-free competition system. Its eigenvalues are negative when $E_{12}$ is stable relatively to infection-free perturbations, i.e. when $\alpha_{12} K_{2}<K_{1}$ and $\alpha_{21} K_{1}<K_{2}$. If the inequalities are reversed, one eigenvalue is positive.

Hence, $E_{12}$ is stable if $\alpha_{12} K_{2}<K_{1}, \alpha_{21} K_{1}<K_{2}$ and $R_{12}<1$. It is unstable if any of these inequalities is reversed.

Remark 3.1 It is useful to distinguish between two subcases when $R_{12}>1$ : in the first, both eigenvalues of TL have positive real parts; in the other, the eigenvalues are real and opposite in sign. In the first subcase, necessarily we have $R_{1}>1$ and $R_{2}>1$. In fact, both eigenvalues have positive real parts if and only $\operatorname{tr}(T L)>0$ and $\operatorname{det}(T L)>0$; these two conditions together imply

$$
\beta_{i i} N_{i E}-\left(b_{i}+\gamma_{i}+\delta_{i}-\frac{a_{i} r_{i}}{K_{i}} N_{i E}\right)>0 \quad(i=1,2)
$$


i.e.

$$
Q_{i}\left(N_{i E}\right):=\frac{\beta_{i i} N_{i E}}{b_{i}+\gamma_{i}+\delta_{i}-\frac{a_{i} r_{i}}{K_{i}} N_{i E}}>1
$$

Since $N_{i E}<K_{i}$ and $Q_{i}(\cdot)$ are increasing functions, we obtain $1<Q_{i}\left(K_{i}\right)=$ $R_{i}$

The results of this section are summarized in the table below.

\begin{tabular}{|c|c|c|}
\hline Equilibrium & Existence? & Stable? \\
\hline$E_{0}=(0,0,0,0)$ & Yes & No \\
\hline$E_{1}=\left(0, K_{1}, 0,0\right)$ & Yes & $\alpha_{21} K_{1}>K_{2}$ and $R_{1}<1$ \\
\hline$E_{2}=\left(0,0,0, K_{2}\right)$ & Yes & $\alpha_{12} K_{2}>K_{1}$ and $R_{2}<1$ \\
\hline$B_{1}=\left(I_{1}^{*}, N_{1}^{*}, J_{2}^{*}, 0\right)$ & $R_{1}>1$ & $\pi_{2}=r_{2}\left(1-\frac{\alpha_{21} N_{1}^{*}}{K_{2}}\right)-\delta_{2} J_{2}^{*}<0$ \\
\hline$B_{2}=\left(J_{1}^{*}, 0, I_{2}^{*}, N_{2}^{*}\right)$ & $R_{2}>1$ & $\pi_{1}=r_{1}\left(1-\frac{\alpha_{12} N_{2}^{*}}{K_{1}}\right)-\delta_{1} J_{1}^{*}<0$ \\
\hline$E_{12}=\left(0, N_{1 E}, 0, N_{2 E}\right)$ & $\alpha_{i j} K_{j}<K_{i}$ (or both reversed) & $\alpha_{i j} K_{j}<K_{i}$ and $R_{12}<1$ \\
\hline
\end{tabular}

Table 1

Conditions for existence and stability of boundary equilibria

\section{Persistence}

We start by recalling some basic definition and results about persistence, following the presentation by Thieme [14].

Let $X$ be a metric space with metric $d$ and the union of two disjoint subsets $X_{1}, X_{2}$, and $\Phi$ a continuous semiflow on $X_{1}$, i.e., a continuous mapping $\Phi$ : 
$[0, \infty) \times X_{1} \longrightarrow X_{1}$ with the following properties:

$$
\Phi_{t} \circ \Phi_{s}=\Phi_{t+s}, t, s \geq 0 ; \quad \Phi_{0}(x)=x, x \in X_{1}
$$

Let $Y_{2}$ be a subset of $X_{2}$, and recall that the distance $d(x, Y)$ of a point $x \in X$ from a subset $Y$ of $X$ is defined by $d(x, Y)=\inf _{y \in Y} d(x, y)$. Then

$Y_{2}$ is called a weak repeller for $X_{1}$ if

$$
\limsup _{t \rightarrow \infty} d\left(\Phi_{t}\left(x_{1}\right), Y_{2}\right)>0 \quad \forall x_{1} \in X_{1}
$$

$Y_{2}$ is called a strong repeller for $X_{1}$ if

$$
\liminf _{t \rightarrow \infty} d\left(\Phi_{t}\left(x_{1}\right), Y_{2}\right)>0 \quad \forall x_{1} \in X_{1}
$$

$Y_{2}$ is called a uniform weak repeller for $X_{1}$ if there is some $\epsilon>0$ such that

$$
\limsup _{t \rightarrow \infty} d\left(\Phi_{t}\left(x_{1}\right), Y_{2}\right)>\epsilon \quad \forall x_{1} \in X_{1}
$$

$Y_{2}$ is called a uniform strong repeller for $X_{1}$ if there is some $\epsilon>0$ such that

$$
\liminf _{t \rightarrow \infty} d\left(\Phi_{t}\left(x_{1}\right), Y_{2}\right)>\epsilon \quad \forall x_{1} \in X_{1}
$$

Let $M$ be a subset of $X, M$ is called forward invariant if and only if $\Phi_{t}(M) \subset$ $M, t>0$, and invariant if and only if $\Phi_{t}(M)=M, t>0$. A compact invariant subset $M$ of $Y \subseteq X$ is called an isolated compact invariant set in $Y$ if there is an open subset $U$ of $X$ such that there is no invariant set $\tilde{M}$ with $M \subseteq \tilde{M} \subseteq U \cap Y$ except $M$. A finite covering $M=\bigcup_{k=1}^{m} M_{k}$ in $X_{2}$ is called isolated if the sets $M_{k}$ are pairwise disjoint subsets of $X_{2}$, which are isolated compact invariant sets in $X$. 
A set $M \subset X_{2}$ is said to be chained (in $X_{2}$ ) to another (not necessarily different) set $N \subset X_{2}$, symbolically $M \mapsto N$, if there is some $y \in X_{2}, y \notin$ $M \cup N$, and a full orbit through $y$ in $X_{2}$ whose $\alpha$-limit set is contained in $M$ and whose $\omega$-limit set is contained in $N$. We recall that an element $y \in X$ has a full orbit, if there is a function $x(t),-\infty<t<\infty$, such that $x(0)=y$ and $x(t+s)=\Phi_{t}(x(s))$ for all $t \geq 0, s \in R$. Moreover, the $\omega$-limit set of a point $y$ is defined as usual:

$$
\omega(y)=\bigcap_{t \geq 0} \overline{\Phi([t, \infty) \times\{y\})}
$$

A finite covering $M=\bigcup_{k=1}^{m} M_{k}$ is called cyclic if, after possible renumbering, $M_{1} \mapsto M_{1}$ or $M_{1} \mapsto M_{2} \mapsto \cdots \mapsto M_{k} \mapsto M_{1}$ for some $k \in\{2,3, \cdots, m\} . M$ is called an acyclic covering otherwise.

We now state two useful results of Thieme [14] on how to obtain persistence through the acyclicity of the flow on $X_{2}$.

Lemma 4.1 ([14, Theorem 4.5]) Let $X$ be locally compact, and let $X_{2}$ be compact in $X$ and $X_{1}$ be forward invariant under the continuous semiflow $\Phi$ on $X$. Assume that

$$
\Omega_{2}=\bigcup_{y \in Y_{2}} \omega(y), \quad Y_{2}=\left\{x \in X_{2} ; \Phi_{t}(x) \in X_{2}, \forall t>0\right\}
$$

has an acyclic isolated covering $M=\bigcup_{k=1}^{m} M_{k}$. If each part $M_{k}$ of $M$ is a weak repeller for $X_{1}$, then $X_{2}$ is a uniform strong repeller for $X_{1}$.

Lemma $4.2\left(\left[14\right.\right.$, Proposition 4.3]) Let $X$ be locally compact, and let $X_{2}$ be compact in $X$ and $X_{1}$ be forward invariant under the continuous semiflow 
$\Phi$ on $X$. Let $x_{n}$ be a sequence of elements in $X_{1}$ satisfying

$$
\limsup _{t \rightarrow \infty} d\left(\Phi_{t}\left(x_{n}\right), X_{2}\right) \rightarrow 0, \quad n \rightarrow \infty
$$

Let $M=\bigcup_{k=1}^{m} M_{k}$ be an isolated covering of $\Omega_{2}$ such that $\omega\left(x_{n}\right) \not \subset M_{k}$ for all $n, k$. Then $M$ is cyclic.

We will use these concepts with $\Phi_{t}$ denoting the mapping given by $\Phi_{t}(x)=$ $\Phi(t, x)$, the solution of system (2.2) with initial condition $x \in X=D=$ $\left\{\left(I_{1}, N_{1}, I_{2}, N_{2}\right) \mid 0 \leq I_{i} \leq 1,0 \leq N_{i} \leq K_{i}, i=1,2\right\}$. We analyze the persistence of system (2.2), meaning that a given set $D_{2} \subset X$ is a repeller for $D_{1}=X \backslash D_{2}$ or some $\tilde{D}_{1} \subset D_{1}$. $D_{2}$ will represent (part of) the boundary of the compact set $D$. For ease of notation, we will let $x(t)=\left(I_{1}(t), N_{1}(t), I_{2}(t), N_{2}(t)\right)=\Phi_{t}\left(x^{0}\right)$ with $x^{0}=\left(I_{1}(0), N_{1}(0), I_{2}(0), N_{2}(0)\right)$.

At first, we consider persistence of the populations.

Theorem 4.3 For system (2.2), we have the following result: there exists an $\epsilon>0$ such that, for any solution $x(t)$ with $N_{1}(0)>0, N_{2}(0)>0$, $\liminf _{t \rightarrow \infty} \max \left\{N_{1}(t), N_{2}(t)\right\}>\epsilon$.

Proof. Define

$$
D_{2}=\left\{\left(I_{1}, N_{1}, I_{2}, N_{2}\right) \mid 0 \leq I_{i} \leq 1, N_{i}=0, i=1,2\right\}, D_{1}=D \backslash D_{2}
$$

$D_{2}$ is clearly compact (in $D$ ), $D_{1}$ and $D_{2}$ are forward invariant.

Let $\Omega_{2}=\bigcup_{y \in D_{2}} \omega(y)$.

It is easy to see that $\Omega_{2}=\left\{E_{0}\right\}=\{(0,0,0,0)\}$ and $\left\{E_{0}\right\}$ is an acyclic covering for $\Omega_{2}$. Looking at system (2.2) we see that this covering is isolated and a weak repeller for $D_{1}$. So by Lemma $4.1, D_{2}$ is a uniform strong repeller for $D_{1}$, i.e. 
the thesis.

The previous result shows that always at least one population does not get extinct. Let now consider assumptions that guarantee strong persistence of one specific host, say host population 1.

Theorem 4.4 Let $\alpha_{12} K_{2}<K_{1}$ and either

a) $R_{2} \leq 1$

or

b) $R_{2}>1$ and $\pi_{1}>0$.

Then there exists an $\epsilon>0$ such that $\liminf _{t \rightarrow \infty} N_{1}(t)>\epsilon$, for any solution $x(t)$ with $N_{1}(0)>0$.

Proof. Define

$$
D_{2}=\left\{\left(I_{1}, N_{1}, I_{2}, N_{2}\right) \mid 0 \leq I_{i} \leq 1, N_{1}=0,0 \leq N_{2} \leq K_{2}\right\}, D_{1}=D \backslash D_{2} .
$$

The proof of case a) is analogous (but simpler) to that of case b). Hence we restrict the analysis to case b).

$D_{2}$ is compact in $D, D_{1}$ and $D_{2}$ are forward invariant. Let $\Omega_{2}=\bigcup_{y \in D_{2}} \omega(y)$.

In order to study $\Omega_{2}$, we analyze the semiflow induced by $(2.2)$ on the forward invariant set $D_{2}$, i.e., for $N_{1} \equiv 0$ :

$$
\left\{\begin{array}{l}
\frac{d I_{1}}{d t}=\left[-\left(b_{1}+\gamma_{1}+\delta_{1}\right)+\delta_{1} I_{1}\right] I_{1}+\beta_{12}\left(1-I_{1}\right) I_{2} N_{2} \\
\frac{d I_{2}}{d t}=\left[\beta_{22}\left(1-I_{2}\right) N_{2}-\left(b_{2}-\frac{a_{2} r_{2} N_{2}}{K_{2}}\right)-\left(\gamma_{2}+\delta_{2}\right)+\delta_{2} I_{2}\right] I_{2} \\
\frac{d N_{2}}{d t}=\left[r_{2}\left(1-\frac{N_{2}}{K_{2}}\right)-\delta_{2} I_{2}\right] N_{2}
\end{array}\right.
$$


It is easy to see that there are three equilibria $E_{0}=(0,0,0,0), E_{2}=\left(0,0,0, K_{2}\right)$ and $B_{2}=\left(J_{1}^{*}, 0, I_{2}^{*}, N_{2}^{*}\right)$ for system (4.2). By Section 2.2 and the first equation of system (4.2), we have: (1) any solution $\left(I_{1}(t), 0, I_{2}(t), N_{2}(t)\right) \in D_{2}$ with $N_{2}(0)=0$ tends to $E_{0}$ as $t \rightarrow \infty ;(2)$ any solution $\left(I_{1}(t), 0, I_{2}(t), N_{2}(t)\right) \in$ $D_{2}$ with $I_{2}(0)=0, N_{2}(0)>0$ tends to $E_{2}$ as $t \rightarrow \infty$; (3) any solution $\left(I_{1}(t), 0, I_{2}(t), N_{2}(t)\right) \in D_{2}$ with $I_{2}(0)>0, N_{2}(0)>0$ tends to $B_{2}$ as $t \rightarrow \infty$. So $\Omega_{2}$ consists of the three equilibria. And it is easy to see that these equilibria cannot be chained to themselves in $D_{2}$. Furthermore, they cannot be chained to each other in a cyclic way in $D_{2}$.

Let $M_{1}=\left\{E_{0}\right\}, M_{2}=\left\{E_{2}\right\}, M_{3}=\left\{B_{2}\right\}$, then $M=\bigcup_{i=1}^{3} M_{i}$ represents an acyclic covering for $\Omega_{2}$. To show that this covering is isolated in $D$ and a weak repeller for $D_{1}$, we analyze the behavior of

$$
\frac{d N_{1}}{d t}=\left[r_{1}\left(1-\frac{N_{1}}{K_{1}}-\frac{\alpha_{12} N_{2}}{K_{1}}\right)-\delta_{1} I_{1}\right] N_{1}, \quad N_{1}(0)>0
$$

when a solution $x(t)$ stays close to $E_{0}, E_{2}$ or $B_{2}$ respectively. If $x(t)$ stays close to $E_{0}$, by (4.3) $N_{1}$ increases exponentially. Similarly, if $x(t)$ stays close to $E_{2}$ or $B_{2}$, by (4.3), and the conditions $\alpha_{12} K_{2}<K_{1}$ or $\pi_{1}=r_{1}\left(1-\frac{\alpha_{12} N_{2}^{*}}{K_{1}}\right)-\delta_{1} J_{1}^{*}>0$, $N_{1}$ increases exponentially too. So the covering $M$ is isolated and each part $M_{i}$ is a weak repeller for $D_{1}$.

Hence by Lemma $4.1, D_{2}$ is a uniform strong repeller for $D_{1}$. Namely there exists an $\epsilon>0$ such that $\liminf _{t \rightarrow \infty} N_{1}(t)>\epsilon$, for any solution $x(t)$ with $N_{1}(0)>0$.

Theorem 4.4 assumes that the equilibrium $E_{2}$ is unstable for the pure competition system (2.3). Without that assumption, we may still prove weak persistence of population 1 . 
Theorem 4.5 For system (2.2), if $\alpha_{12} K_{2}>K_{1}, R_{2}>1$ and $\pi_{1}>0$, then we have:

(1) there exists a solution $x(t)=\left(0, N_{1}(t), 0, N_{2}(t)\right)$ with $N_{1}(0)>0$, such that $\lim _{t \rightarrow \infty} N_{1}(t)=0 ;$

(2) there exists an $\epsilon>0$ such that $\limsup _{t \rightarrow \infty} N_{1}(t)>\epsilon$, for any solution $x(t)$ with $I_{1}(0)>0, N_{1}(0)>0, I_{2}(0)>0, N_{2}(0)>0$.

Proof. 1. When $\alpha_{12} K_{2}>K_{1}$, by Section $2.1\left(0, K_{2}\right)$ is stable for system (2.3). So we can find a solution $\left(0, N_{1}(t), 0, N_{2}(t)\right)$ with $N_{1}(0)>0$, such that $\lim _{t \rightarrow \infty} N_{1}(t)=0$.

\section{Define}

$$
\begin{gathered}
D_{2}=\left\{\left(I_{1}, N_{1}, I_{2}, N_{2}\right) \mid 0 \leq I_{i} \leq 1, N_{1}=0,0 \leq N_{2} \leq K_{2}\right\}, D_{1}=D \backslash D_{2}, \\
\tilde{D}_{1}=\left\{\left(I_{1}, N_{1}, I_{2}, N_{2}\right) \mid 0<I_{i} \leq 1,0<N_{i} \leq K_{i}, i=1,2\right\}
\end{gathered}
$$

It is easy to see that $D_{1}, \tilde{D}_{1}$ and $D_{2}$ are forward invariant.

Let $\Omega_{2}=\bigcup_{y \in D_{2}} \omega(y)$.

In order to study $\Omega_{2}$, we must analyze the semiflow on $D_{2}$, i.e. (4.2). As above, $\Omega_{2}$ consists of three equilibria $E_{0}, E_{2}$ and $B_{2}$. And it is easy to see that these equilibria cannot be chained to themselves or to each other in a cyclic way in $D_{2}$.

Letting again $M_{1}=\left\{E_{0}\right\}, M_{2}=\left\{E_{2}\right\}, M_{3}=\left\{B_{2}\right\}$, then $M=\bigcup_{i=1}^{3} M_{i}$ represents an acyclic covering for $\Omega_{2}$, and it is easy to see that this covering is isolated in $D$ and each part $M_{i}$ is a weak repeller for $\tilde{D}_{1}$.

We cannot apply Lemma 4.1, since it is not true that each part is a weak 
repeller for $D_{1}$. However, we will show, using Lemma 4.2, that $D_{2}$ is a uniform weak repeller for $\tilde{D}_{1}$.

If $D_{2}$ is not a uniform weak repeller for $\tilde{D}_{1}$, then we find a sequence $x_{n}=$ $\left(I_{1 n}, N_{1 n}, I_{2 n}, N_{2 n}\right) \in \tilde{D}_{1} \subset D_{1}$ satisfying

$$
\limsup _{t \rightarrow \infty} d\left(\Phi_{t}\left(x_{n}\right), D_{2}\right) \rightarrow 0, \quad n \rightarrow \infty
$$

As each part $M_{i}$ is a weak repeller for $\tilde{D}_{1}$, we have $\omega\left(x_{n}\right) \not \subset M_{i}$ for all $n, i$. Hence the assumptions of Lemma 4.2 are satisfied (indeed $D_{2}$ is compact) and the covering $M$ has to be cyclic, in contradiction to the acyclic property of $M$. So $D_{2}$ is a uniform weak repeller for $\tilde{D}_{1}$, which is the thesis.

The previous Theorem shows that a species that would get extinct without the infection, may (weakly) persist in presence of the infection.

Remark 4.6 The results on the stability of the boundary equilibria yield conditions for the non-persistence of $N_{1}$. Precisely, if $\alpha_{12} K_{2}>K_{1}$ and $R_{2}<1$, or if $R_{2}>1$ and $\pi_{1}<0$, then $N_{1}$ is not persistent. In fact, in the first case, $E_{2}$ is asymptotically stable; in the second, $B_{2}$ is asymptotically stable.

Clearly, similar results would hold for species 2, exchanging all indices.

We now consider persistence of the infection.

Theorem 4.7 If $\alpha_{12} K_{2}>K_{1}, \alpha_{21} K_{1}<K_{2}$ and $R_{2}>1$, there exists an $\epsilon>0$ such that, for any solution $x(t)$ with $N_{1}(0)>0, N_{2}(0)>0$ and $I_{2}(0)>0$ or $I_{1}(0)>0, \liminf _{t \rightarrow \infty} \min \left\{I_{1}(t), I_{2}(t)\right\}>\epsilon$.

Proof. Define

$$
D_{2}=\left\{\left(I_{1}, N_{1}, I_{2}, N_{2}\right) \mid I_{1}=0 \text { or } I_{2}=0,0 \leq N_{i} \leq K_{i}\right\}, D_{1}=D \backslash D_{2},
$$




$$
\tilde{D}_{1}=\left\{\left(I_{1}, N_{1}, I_{2}, N_{2}\right) \mid 0<I_{i} \leq 1,0<N_{i} \leq K_{i}, i=1,2\right\}
$$

It is easy to see that $D_{1}$ and $\tilde{D}_{1}$ are forward invariant.

From the assumption $N_{1}(0)>0, N_{2}(0)>0$, and $I_{2}(0)>0$ or $I_{1}(0)>0$, it follows that $\Phi_{t}\left(x^{0}\right) \in \tilde{D}_{1}$ for all $t>0$. We can then assume $x_{0} \in \tilde{D}_{1}$.

Let

$$
\Omega_{2}=\bigcup_{y \in Y_{2}} \omega(y), \quad Y_{2}=\left\{x=\left(I_{1}, N_{1}, I_{2}, N_{2}\right) \in D_{2} ; \Phi_{t}(x) \in D_{2}, \forall t>0\right\}
$$

By analyzing the semiflow induced by $(2.2)$, we find that $\Omega_{2}$ consists of three equilibria, $E_{0}=(0,0,0,0), E_{1}=\left(0, K_{1}, 0,0\right)$ and $E_{2}=\left(0,0,0, K_{2}\right)$. Furthermore, they cannot be chained to themselves, or to each other in a cyclic way in $D_{2}$.

Setting $M_{1}=\left\{E_{0}\right\}, M_{2}=\left\{E_{1}\right\}, M_{3}=\left\{E_{2}\right\}, M=\bigcup_{i=1}^{3} M_{i}$ represents an acyclic covering for $\Omega_{2}$. By analysing the flow in the neighbourhood of each equilibrium, it is easy to see that this covering is isolated in $D$ and each part $M_{i}$ is a weak repeller for $\tilde{D}_{1}$. As in the Proof of Theorem 4.5, we can use this to prove that $D_{2}$ is a uniform weak repeller for $\tilde{D}_{1}$, i.e. there exists an $0<\tilde{\epsilon}<\min \left\{J_{1}^{*}, J_{2}^{*}, I_{1}^{*}, I_{2}^{*}\right\}$ such that

$$
\limsup _{t \rightarrow \infty} \min \left\{I_{1}(t), I_{2}(t)\right\}>\tilde{\epsilon}
$$

for any solution $x(t)$ with $N_{1}(0)>0, N_{2}(0)>0, I_{1}(0)>0$ and $I_{2}(0)>0$.

Now, we prove that $D_{2}$ is a uniform strong repeller for $\tilde{D}_{1}$. Suppose that $D_{2}$ is not a uniform strong repeller for $\tilde{D}_{1}$. Then there exist sequences $x_{j}^{0}=$ 
$\left(I_{1}^{j}(0), N_{1}^{j}(0), I_{2}^{j}(0), N_{2}^{j}(0)\right) \in \tilde{D}_{1}$ and $0<\epsilon_{j}<\tilde{\epsilon}$ such that

$$
\liminf _{t \rightarrow \infty} \min \left\{I_{1}^{j}(t), I_{2}^{j}(t)\right\}<\epsilon_{j} \quad \text { for } j=1,2, \ldots
$$

Here $\lim _{t \rightarrow \infty} \epsilon_{j}=0$ and $\left(I_{1}^{j}(t), N_{1}^{j}(t), I_{2}^{j}(t), N_{2}^{j}(t)\right)$ are the solutions of system (2.2) with initial values $x_{j}^{0} \in \tilde{D}_{1}$.

From (4.5) and (4.4), we can find sequences $0<r_{j}<s_{j}<t_{j}$ with $\lim _{j \rightarrow \infty} r_{j}=\infty$ such that

$$
\begin{gathered}
\lim _{j \rightarrow \infty} \min \left\{I_{1}^{j}\left(s_{j}\right), I_{2}^{j}\left(s_{j}\right)\right\}=0, \\
\min \left\{I_{1}^{j}\left(r_{j}\right), I_{2}^{j}\left(r_{j}\right)\right\}=\min \left\{I_{1}^{j}\left(t_{j}\right), I_{2}^{j}\left(t_{j}\right)\right\}=\tilde{\epsilon}, \\
\min \left\{I_{1}^{j}(t), I_{2}^{j}(t)\right\} \leq \tilde{\epsilon} \text { for } r_{j} \leq t \leq t_{j} .
\end{gathered}
$$

Moreover, $r_{j}$ may be chosen large enough such that: (1) when $R_{1} \leq 1, N_{2}^{j}(t)>$ $\epsilon^{*}$ for $t \geq r_{j}$, where $\epsilon^{*}>0$ is a positive constant (this follows from Theorem 4.4, reversing the indices); (2) when $R_{1}>1, \max \left\{N_{1}^{j}(t), N_{2}^{j}(t)\right\}>\epsilon^{*}$ for $t \geq r_{j}$ (this follows from Theorem 4.3).

After choosing a subsequence, the sequence $\left(I_{1}^{j}\left(r_{j}\right), N_{1}^{j}\left(r_{j}\right), I_{2}^{j}\left(r_{j}\right), N_{2}^{j}\left(r_{j}\right)\right)$ is convergent in $D$, and let

$\lim _{j \rightarrow \infty}\left(I_{1}^{j}\left(r_{j}\right), N_{1}^{j}\left(r_{j}\right), I_{2}^{j}\left(r_{j}\right), N_{2}^{j}\left(r_{j}\right)\right)=\left(I_{1}^{* *}(0), N_{1}^{* *}(0), I_{2}^{* *}(0), N_{2}^{* *}(0)\right)=x^{* *}(0)$.

Then by $(4.7)$ we have $\min \left\{I_{1}^{* *}(0), I_{2}^{* *}(0)\right\}=\tilde{\epsilon}$, so that $x^{* *}(0) \in D_{1}$.

In the following, we prove the thesis in two steps.

First, we prove by contradiction that $t_{j}-r_{j}$ is unbounded. Suppose in fact that $t_{j}-r_{j}$ is bounded; then, after taking a subsequence, $s_{j}-r_{j}$ is convergent 
and let $\lim _{j \rightarrow \infty}\left(s_{j}-r_{j}\right)=s^{*}$. By the basic properties of flow we have

$$
\lim _{j \rightarrow \infty}\left(I_{1}^{j}\left(r_{j}+s^{*}\right), N_{1}^{j}\left(r_{j}+s^{*}\right), I_{2}^{j}\left(r_{j}+s^{*}\right), N_{2}^{j}\left(r_{j}+s^{*}\right)\right)=x^{* *}\left(s^{*}\right)
$$

where $x^{* *}(t)$ is the solution of system $(2.2)$ with initial value $x^{* *}(0)$. Since $x^{* *}(0) \in D_{1}$ and $D_{1}$ is invariant, we have $x^{* *}\left(s^{*}\right) \in D_{1}$. At the same time, by the basic properties of flow we also have

$$
\lim _{j \rightarrow \infty}\left(I_{1}^{j}\left(s_{j}\right), N_{1}^{j}\left(s_{j}\right), I_{2}^{j}\left(s_{j}\right), N_{2}^{j}\left(s_{j}\right)\right)=x^{* *}\left(s^{*}\right) .
$$

Moreover we have $x^{* *}\left(s^{*}\right) \in D_{2}$ by (4.6) and the compactness of $D_{2}$. A contradiction occurs, so $t_{j}-r_{j}$ is unbounded.

Next, we prove a contradiction under the assumption of non-uniform strong repeller. If $x^{* *}(0) \in \tilde{D}_{1}$, then, by (4.4), we have

$$
\limsup _{t \rightarrow \infty} \min \left\{I_{1}^{* *}(t), I_{2}^{* *}(t)\right\}>\tilde{\epsilon}
$$

We show that (4.10) always holds. If $x^{* *}(0) \in D_{1} \backslash \tilde{D}_{1}$, then we have two cases:

(1) when $R_{1} \leq 1$, we have seen above that $N_{2}^{j}(t)>\epsilon^{*}$ for $t \geq r_{j}$ so that $N_{2}^{* *}(0) \geq \epsilon^{*}$; then, from $(4.7)$, we have $\min \left\{I_{1}^{* *}(0), I_{2}^{* *}(0)\right\}=\tilde{\epsilon}$, so that it must be $N_{1}^{* *}(0)=0$. Then $N_{1}^{* *}(t) \equiv 0$ and from Lemma 2.2 and the first equation of system (2.2) we have

$$
\lim _{t \rightarrow \infty} I_{1}^{* *}(t)=J_{1}^{*} \text { and } \lim _{t \rightarrow \infty} I_{2}^{* *}(t)=I_{2}^{*}
$$

which means that (4.10) holds.

(2) when $R_{1}>1$, we obtain by $\max \left\{N_{1}^{j}(t), N_{2}^{j}(t)\right\}>\epsilon^{*}$ for $t \geq r_{j}$ that either $N_{1}^{* *}(0)=0$ and $N_{2}^{* *}(0) \geq \epsilon^{*}$ or vice versa. Analogously to the previous case, we can then prove that (4.10) holds.

Now, since $t_{j}-r_{j}$ is unbounded, after choosing a subsequence, we may assume 
that $t_{j}-r_{j}$ is increasing monotonically and $\lim _{j \rightarrow \infty} t_{j}-r_{j}=\infty$. Then by (4.8) we have, for $k>j$,

$$
\min \left\{I_{1}^{k}\left(r_{k}+r\right), I_{2}^{k}\left(r_{k}+r\right)\right\} \leq \tilde{\epsilon} \text { for } 0 \leq r \leq t_{j}-r_{j}
$$

Fixing $r$ and $j$, and letting $k$ tend to infinity, we obtain, for $0 \leq r \leq t_{j}-r_{j}$,

$$
\min \left\{I_{1}^{* *}(r), I_{2}^{* *}(r)\right\}=\lim _{k \rightarrow \infty} \min \left\{I_{1}^{k}\left(r_{k}+r\right), I_{2}^{k}\left(r_{k}+r\right)\right\} \leq \tilde{\epsilon}
$$

We now let $j \rightarrow \infty$ and, as $\lim _{j \rightarrow \infty} t_{j}-r_{j}=\infty$, (4.11) holds for all $r \geq 0$, contradicting (4.10). This means that $D_{2}$ is a uniform strong repeller for $\tilde{D}_{1}$, i.e. the thesis.

A similar result holds exchanging hosts 1 and 2 .

For the cases where the competition model has an internal equilibrium, we have the following:

Theorem 4.8 For system (2.2), if $\alpha_{12} K_{2}<K_{1}, \alpha_{21} K_{1}<K_{2}$ and $R_{12}>1$, there exists an $\epsilon>0$ such that $\liminf _{t \rightarrow \infty} \min \left\{I_{1}(t), I_{2}(t)\right\}>\epsilon$, for any solution $x(t)$ with $N_{1}(0)>0, N_{2}(0)>0$ and $I_{2}(0)>0$ or $I_{1}(0)>0$.

Proof. Define

$$
\begin{gathered}
D_{2}=\left\{\left(I_{1}, N_{1}, I_{2}, N_{2}\right) \mid I_{1}=0 \text { or } I_{2}=0,0 \leq N_{i} \leq K_{i}\right\}, D_{1}=D \backslash D_{2}, \\
\tilde{D}_{1}=\left\{\left(I_{1}, N_{1}, I_{2}, N_{2}\right) \mid 0<I_{i} \leq 1,0<N_{i} \leq K_{i}, i=1,2\right\}
\end{gathered}
$$

$D_{1}$ and $\tilde{D}_{1}$ are forward invariant. Let

$$
\Omega_{2}=\bigcup_{y \in Y_{2}} \omega(y), \quad Y_{2}=\left\{x=\left(I_{1}, N_{1}, I_{2}, N_{2}\right) \in D_{2} ; \Phi_{t}(x) \in D_{2}, \forall t>0\right\}
$$

By analyzing the semiflow induced by (2.2), we find that $\Omega_{2}$ consists of four equilibria, $E_{0}=(0,0,0,0), E_{1}=\left(0, K_{1}, 0,0\right), E_{2}=\left(0,0,0, K_{2}\right)$ and $E_{12}=$ 
$\left(0, N_{1 E}, 0, N_{2 E}\right)$. These equilibria cannot be chained to themselves in $D_{2}$ or to each other in a cyclic way in $D_{2}$.

Let $M_{1}=\left\{E_{0}\right\}, M_{2}=\left\{E_{1}\right\}, M_{3}=\left\{E_{2}\right\}, M_{4}=\left\{E_{12}\right\}$; then $M=\bigcup_{i=1}^{4} M_{i}$ represents an acyclic covering for $\Omega_{2}$. To show that this covering is isolated in $D$, we analyze the behavior of system (2.2) when any solution $x(t)$ stays close to $E_{0}, E_{1}, E_{2}$ or $E_{12}$ respectively.

If $x(t)$ stays close to $E_{0}$, we have two cases: (1) if $N_{1}(0)=N_{2}(0)=0$, then $N_{1}(t)=N_{2}(t) \equiv 0$. In this case, $I_{1}$ and $I_{2}$ decrease exponentially; (2) if $N_{1}(0)>0$ or $N_{2}(0)>0$, then $N_{1}(t)>0$ or $N_{2}(t)>0$. By $(2.2) N_{1}$ or $N_{2}$ increases exponentially. So by the two cases, $M_{1}$ is isolated in $D$.

If $x(t)$ stays in a small neighbourhood of $E_{2}$, we have three cases:

(1) if $N_{1}(0)=I_{2}(0)=0$, then $N_{1}(t)=I_{2}(t) \equiv 0$. By $(2.2), K_{2}-N_{2}(t)$ decreases exponentially, so $x(t)$ has to exit the neighbourhood as $t \rightarrow$ $-\infty$

(2) if $N_{1}(0)>0$, then $N_{1}(t)>0$ and

$$
\frac{d N_{1}}{d t} \geq\left[r_{1}\left(1-\frac{\eta}{K_{1}}-\frac{\alpha_{12} K_{2}}{K_{1}}\right)-\delta_{1} \eta\right] N_{1}
$$

where $\eta$ is the size of the neighbourhood. Since $\alpha_{12} K_{2}<K_{1}$, choosing $\eta>0$ small enough, we see that $N_{1}$ increases exponentially as $t \rightarrow+\infty$;

(3) if $N_{1}(0)=0$ and $I_{2}(0)>0$, then $N_{1}(t) \equiv 0$ and $I_{2}(t)>0$ for all $t>$ 0. In this case, $\left(I_{2}(t), N_{2}(t)\right)$ satisfy system (2.4) that has no invariant subset other than $E_{2}$ in its neighbourhood; in fact, if $R_{2} \leq 1, I_{2}(t)$ is monotonically decreasing; otherwise $I_{2}(t)$ increases to $I_{2}^{*}$.

So by all cases, $M_{3}$ is isolated in $D$. 
Similarly, we can prove that $M_{2}$ is isolated in $D$.

If $x(t)$ stays close to $E_{12}$, we have two cases:

(1) if $I_{1}(0)=I_{2}(0)=0$, then $I_{1}(t)=I_{2}(t) \equiv 0$. Then (2.3) with $\alpha_{12} K_{2}<$ $K_{1}$ and $\alpha_{21} K_{1}<K_{2}$ shows that $\left(N_{1}(t), N_{2}(t)\right)$ goes far away from $E_{12}$ as $t \rightarrow-\infty$

(2) if $I_{1}(0)>0$ or $I_{2}(0)>0$, then $I_{1}(t)>0$ and $I_{2}(t)>0$ for all $t>0$. When $x(t)$ stays very close to $E_{12}$, by $(2.2)$ there exists some $\delta>0$ (related to the size of the neighbourhood of $E_{12}$ ) such that

$$
\left\{\begin{array}{l}
\frac{d I_{1}}{d t}>\tilde{c}_{11} I_{1}+\tilde{c}_{12} I_{2} \\
\frac{d I_{2}}{d t}>\tilde{c}_{21} I_{1}+\tilde{c}_{22} I_{2}
\end{array}\right.
$$

where $\tilde{c}_{i j}=c_{i j}-\delta$ and $c_{i j}$ are the entries of the top-left matrix $T L$ of the matrix $C_{12}$ in (3.3). By choosing $\delta$ small enough, the matrix $\tilde{C}$ in (4.12) has positive non-diagonal elements and its largest eigenvalue is positive, since $R_{12}>1$ [17]. Hence the solution of the linear quasi-monotonic system

$$
\left\{\begin{array}{l}
\frac{d x_{1}}{d t}=\tilde{c}_{11} x_{1}+\tilde{c}_{12} x_{2} \\
\frac{d x_{2}}{d t}=\tilde{c}_{21} x_{1}+\tilde{c}_{22} x_{2}
\end{array}\right.
$$

with $x_{1}(0)>0, x_{2}(0)>0$ are exponentially increasing as $t \rightarrow \infty$. By the comparison principle, $\left(I_{1}(t), I_{2}(t)\right)$ goes away from $(0,0)$.

Hence, by the two cases $M_{4}$ is isolated in $D$, so that $M$ is isolated in $D$.

The arguments used in these proofs (cases 2.) show also that each part $M_{i}$ is a weak repeller for $\tilde{D}_{1}$. 
Using Lemma 4.2 as in the proof of Theorem 4.5, we can prove that $D_{2}$ is a uniform weak repeller for $\tilde{D}_{1}$; and then as in the proof of Theorem 4.7, we can prove that $D_{2}$ is a uniform strong repeller for $\tilde{D}_{1}$, i.e. the thesis.

The previous Theorem shows that it is possible that an infection that would die out in either species without the inter-infection of disease, may persist in both species in presence of this factor.

By the strong uniform persistence of infection, in several cases, the weak uniform persistence of populations (Theorem 4.5) can be strengthened to the strong uniform persistence.

Theorem 4.9 Let $\alpha_{12} K_{2}>K_{1}, R_{2}>1, \pi_{1}>0$ and either

a) $\alpha_{21} K_{1}<K_{2}$

or

b) $\alpha_{21} K_{1}>K_{2}, R_{1}>1$ and $R_{12}>1$.

Then there exists an $\epsilon>0$ such that $\liminf _{t \rightarrow \infty} N_{1}(t)>\epsilon$, for any solution $x(t)$ with $N_{1}(0)>0, N_{2}(0)>0$ and $I_{1}(0)>0$ or $I_{2}(0)>0$.

Proof. The idea and method of the proof are similar to those of the previous Theorem and the proof is, therefore, omitted.

A similar result holds exchanging hosts 1 and 2 .

Finally, by the strong uniform persistence of populations and infection, we can obtain the strong uniform persistence of (2.2) relatively to all components.

Theorem 4.10 For system (2.2), if $\alpha_{12} K_{2}>K_{1}, \alpha_{21} K_{1}<K_{2}, R_{2}>1$, 
$\pi_{1}>0$, and either (1) $R_{1}<1$, or (2) $R_{1}>1$ and $\pi_{2}>0$, then there exists an $\epsilon>0$ such that, for any solution $x(t)$ with $N_{1}(0)>0, N_{2}(0)>0$ and $I_{1}(0)>0$ or $I_{2}(0)>0, \liminf _{t \rightarrow \infty} \min \left\{I_{1}(t), N_{1}(t), I_{2}(t), N_{2}(t)\right\}>\epsilon$.

A similar result holds exchanging hosts 1 and 2 .

For the cases where the competition model has an internal equilibrium, we have the following:

Theorem 4.11 For system (2.2), if $\alpha_{12} K_{2}<K_{1}, \alpha_{21} K_{1}<K_{2}, R_{12}>1$, and any one of the following conditions holds:

(1) $R_{1}<1, R_{2}<1$;

(2) $R_{1}>1, R_{2}<1, \pi_{2}>0$;

(3) $R_{1}<1, R_{2}>1, \pi_{1}>0$;

(4) $R_{1}>1, R_{2}>1, \pi_{1}>0, \pi_{2}>0$,

\begin{tabular}{|c|c|c|c|c|c|c|}
\hline \multicolumn{6}{|c|}{ conditions } & conclusion \\
\hline$R_{j}<1$ & \multicolumn{5}{|c|}{$\alpha_{i j} K_{j}>K_{i}$} & $\mathrm{NP}, E_{j}$ stable \\
\hline$(j \neq i, j=1,2)$ & \multicolumn{5}{|c|}{$\alpha_{i j} K_{j}<K_{i}$} & SUP \\
\hline \multirow{6}{*}{$R_{j}>1$} & \multicolumn{5}{|c|}{$\pi_{i}<0$} & NP, $B_{j}$ stable \\
\hline & \multirow{5}{*}{$\pi_{i}>0$} & \multicolumn{4}{|c|}{$\alpha_{i j} K_{j}<K_{i}$} & SUP \\
\hline & & \multirow{4}{*}{$\alpha_{i j} K_{j}>K_{i}$} & \multicolumn{3}{|c|}{$\alpha_{j i} K_{i}<K_{j}$} & SUP \\
\hline & & & \multirow{3}{*}{$\alpha_{j i} K_{i}>K_{j}$} & \multicolumn{2}{|c|}{$R_{i}<1$} & WUP \\
\hline & & & & \multirow[t]{2}{*}{$R_{i}>1$} & $R_{12}<1$ & WUP \\
\hline & & & & & $R_{12}>1$ & SUP \\
\hline
\end{tabular}

Table 2

Persistence of either population $N_{i}(i=1,2)$, where, NP stands for non-persistence, SUP stands for strong uniform persistence, WUP stands for weak uniform persistence 
then there exists an $\epsilon>0$ such that $\liminf _{t \rightarrow \infty} \min \left\{I_{1}(t), N_{1}(t), I_{2}(t), N_{2}(t)\right\}>\epsilon$, for any solution $x(t)$ with $N_{1}(0)>0, N_{2}(0)>0$ and $I_{1}(0)>0$ or $I_{2}(0)>0$.

Remark 4.12 Note that, by Remark 3.1, if both eigenvalues of the top-left matrix TL of (3.3) have positive real parts, then $R_{1}>1$ and $R_{2}>1$, so that only case 4) may occur, and one needs only check $\pi_{1}>0$ and $\pi_{2}>0$.

Theorem 4.13 For system (2.2), if $\alpha_{12} K_{2}>K_{1}, \alpha_{21} K_{1}>K_{2}, R_{12}>$ 1, $R_{1}>1, R_{2}>1, \pi_{1}>0, \pi_{2}>0$, then there exists an $\epsilon>0$ such that, for any solution $x(t)$ with $N_{1}(0)>0, N_{2}(0)>0$ and $I_{1}(0)>0$ or $I_{2}(0)>0$, $\liminf _{t \rightarrow \infty} \min \left\{I_{1}(t), N_{1}(t), I_{2}(t), N_{2}(t)\right\}>\epsilon$.

\begin{tabular}{|c|c|c|c|c|}
\hline \multicolumn{4}{|c|}{ conditions } & conclusion \\
\hline \multirow[t]{2}{*}{$\alpha_{12} K_{2}>K_{1}, \alpha_{21} K_{1}<K_{2}$} & \multicolumn{3}{|c|}{$R_{2}<1$} & $\mathrm{NP}, E_{2}$ stable \\
\hline & \multicolumn{3}{|c|}{$R_{2}>1$} & SUP \\
\hline \multirow[t]{2}{*}{$\alpha_{12} K_{2}<K_{1}, \alpha_{21} K_{1}>K_{2}$} & \multicolumn{3}{|c|}{$R_{1}<1$} & NP, $E_{1}$ stable \\
\hline & \multicolumn{3}{|c|}{$R_{1}>1$} & SUP \\
\hline \multirow[t]{2}{*}{$\alpha_{12} K_{2}<K_{1}, \alpha_{21} K_{1}<K_{2}$} & \multicolumn{3}{|c|}{$R_{12}<1$} & $\mathrm{NP}, E_{12}$ stable \\
\hline & \multicolumn{3}{|c|}{$R_{12}>1$} & SUP \\
\hline \multirow{4}{*}{$\alpha_{12} K_{2}>K_{1}, \alpha_{21} K_{1}>K_{2}$} & \multicolumn{3}{|c|}{$R_{2}<1$} & $\mathrm{NP}, E_{2}$ stable \\
\hline & \multirow{3}{*}{$R_{2}>1$} & \multicolumn{2}{|c|}{$R_{1}<1$} & $\mathrm{NP}, E_{1}$ stable \\
\hline & & \multirow[t]{2}{*}{$R_{1}>1$} & $R_{12}<1$ & $\mathrm{NP}, 3 \mathrm{~d}-\mathrm{SM}$ at $E_{12}$ \\
\hline & & & $R_{12}>1$ & SUP \\
\hline
\end{tabular}

\section{Table 3}

Persistence of both of $I_{i}(i=1,2)$ where, 3d-SM stands for a 3-dimensional stable manifold

Remark 4.14 If $\alpha_{12} K_{2}<K_{1}, \alpha_{21} K_{1}<K_{2}$ and $R_{12}<1$, then the equilibrium $E_{12}$ is stable; hence system (2.2) is not persistent. 
On the other hand, if $\alpha_{12} K_{2}>K_{1}, \alpha_{21} K_{1}>K_{2}$ and $R_{12}<1$, the equilibrium $E_{12}$ has a 3-dimensional stable manifold $V^{s}$ and a 1-dimensional unstable manifold $V^{u}$ lying on the plane $\left(N_{1}, N_{2}\right)$. Letting

$$
D_{1}=\left\{\left(N_{1}, N_{2}, I_{1}, I_{2}\right): I_{1}>0, I_{2}>0\right\}
$$

it is clear that $D_{2}=D \backslash D_{1}$ is not a repeller for $D_{1}$, since points lying in $D_{1} \cap V^{s}$ are attracted to $E_{12}$. The question of what happens if $x(0) \in D_{1} \backslash V^{s}$ remains open.

We summarize the results on persistence, together with the stability results of the previous Section, in Tables 2, 3 and 4. In reading Table 4, remember that in case III when $R_{12}>1$ and both eigenvalues of the top-left matrix $T L$ of the matrix $C_{12}$ in (3.3) have positive real parts, necessarily $R_{1}>1$ and $R_{2}>1$.

Corollary 4.15 In all conditions listed in Table 4 that guaranteed strong uniform persistence, there exists at least one internal equilibrium of (2.2).

Proof. It follows from a general result from persistence theory [21, Remark 3.10 and Theorem 4.7], [22, pp. 160-166].

\section{$5 \quad$ Bifurcation Phenomena}

In this section, we present, with the help of numerical software, some bifurcation diagrams of system (2.2) in order to explore the possible behaviors of the solutions.

First, we recall the conditions for Hopf bifurcation, following the presentation 


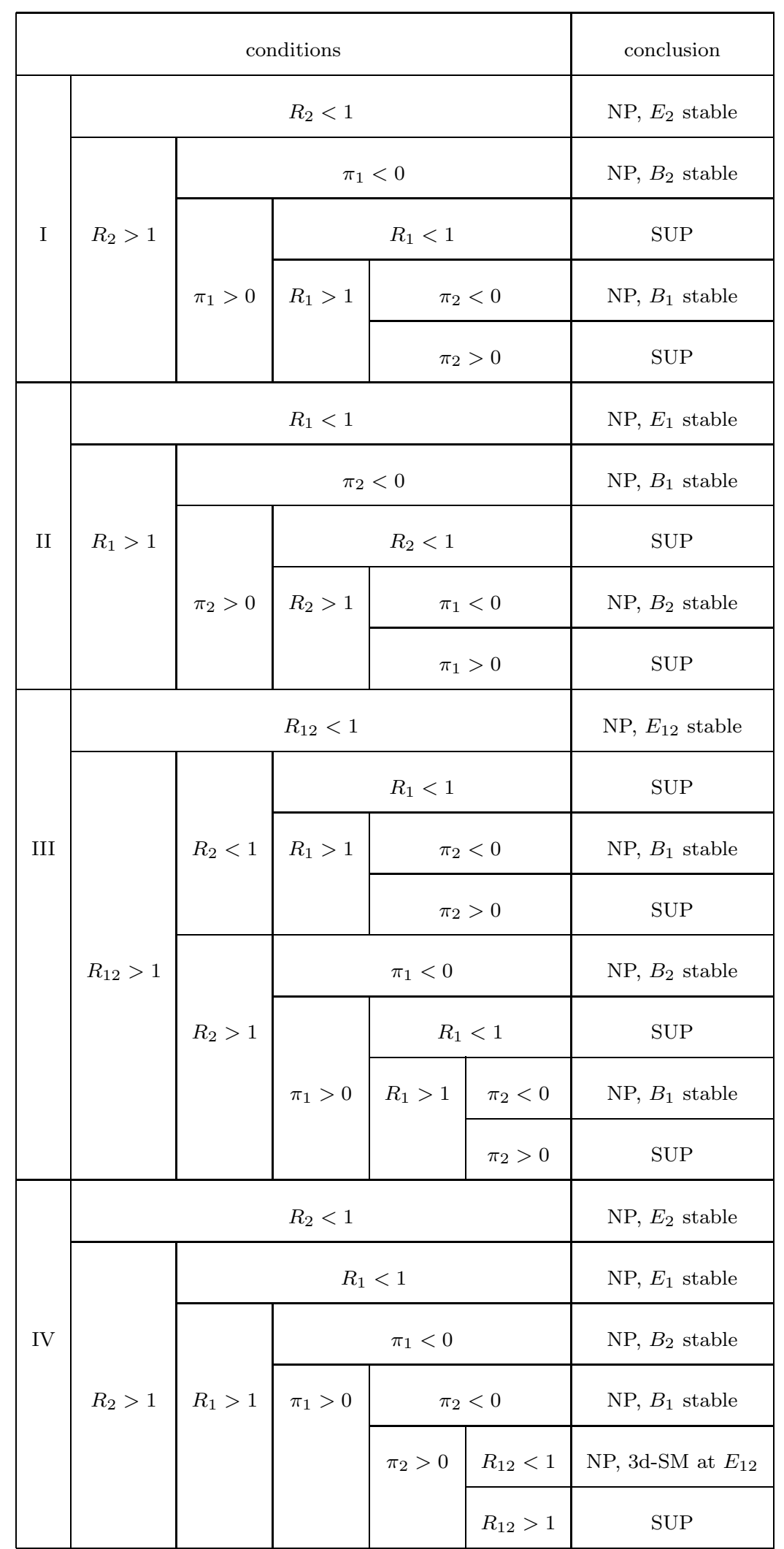

\section{Table 4}

Persistence of all of $I_{1}, N_{1}, I_{2}$ and $N_{2}$. I stands for $\alpha_{12} K_{2}>K_{1}, \alpha_{21} K_{1}<K_{2}$, II stands for $\alpha_{12} K_{2}<K_{1}, \alpha_{21} K_{1}>K_{2}$, III stands for $\alpha_{12} K_{2}<K_{1}, \alpha_{21} K_{1}<K_{2}$, IV stands for $\alpha_{12} K_{2}>K_{1}, \alpha_{21} K_{1}>K_{2} . \quad 34$ 
by Shen and Jing [23].

Consider the following differential system

$$
\frac{d x}{d t}=f(x, \mu), \quad x \in R^{n}, \quad \mu \in R
$$

with an equilibrium point $x=x_{0}(\mu)$. If the Jacobian matrix

$$
A(\mu)=D_{x} f\left(x_{0}(\mu), \mu\right)
$$

has a pair of complex conjugate eigenvalues

$$
\lambda_{1}(\mu) \pm i \lambda_{2}(\mu)
$$

and for some value $\mu=\mu_{0}$,

$$
\lambda_{1}\left(\mu_{0}\right)=0, \quad \lambda_{2}\left(\mu_{0}\right)>0, \quad \frac{d \lambda_{1}\left(\mu_{0}\right)}{d \mu} \neq 0
$$

and the remaining eigenvalues of $A\left(\mu_{0}\right)$ have nonzero real parts, then the crucial hypotheses of Hopf bifurcation theorem are satisfied, and the equilibrium $x_{0}$ bifurcates into a "small amplitude" periodic solution as $\mu$ passes through $\mu_{0}$.

For system (5.1), the characteristic equation at the equilibrium $x=x_{0}$ can be written as

$$
P(\lambda)=\lambda^{n}+c_{n-1}(\mu) \lambda^{n-1}+\cdots+c_{0}(\mu)=0
$$

Conditions (5.2) and (5.3) can be written in terms of the coefficients of (5.4), as stated in the following lemma:

Lemma 5.1 ([23, Theorem 3]) Conditions (5.2) and (5.3) for the existence of the Hopf bifurcation are satisfied for system (5.4), if the following conditions are satisfied: 1) $\mu=\mu_{0}$ is a zero of $H_{n-1}(\mu)=0$; 2) $H_{n-2}\left(\mu_{0}\right) \neq$ 
$0, H_{n-3}\left(\mu_{0}\right) \neq 0, c_{j}\left(\mu_{0}\right)>0, j=0,1,2, \cdots, n-1$; 3) $\frac{d H_{n-1}\left(\mu_{0}\right)}{d \mu} \neq 0$, where $H_{j}(\mu), j=1,2, \cdots, n$, are the Hurwitz determinants.

To use this theorem for system (2.2), we need to discuss Hopf bifurcation at an internal equilibrium. However, for system (2.2) there are no explicit formulae for an internal equilibrium and, in general, not even its existence can be proved. Hence we will study the bifurcation in the very special case, where all the analogous parameters are same for species 1 and 2. Although it is a very particular case, it displays several interesting behaviors that can shed light also outside of this special structure.

Namely, we let

$$
\begin{gathered}
r_{1}=r_{2}=r, b_{1}=b_{2}=b, d_{1}=d_{2}=d, a_{1}=a_{2}=a, K_{1}=K_{2}=K, \\
\alpha_{12}=\alpha_{21}=\alpha, \gamma_{1}=\gamma_{2}=\gamma, \delta_{1}=\delta_{2}=\delta, \beta_{11}=\beta_{22}=\beta_{1}, \beta_{12}=\beta_{21}=\beta_{2} .
\end{gathered}
$$

Then system (2.2) becomes a symmetric (with respect to the exchange of 1 and 2) system.

When $R^{*}>1$, there exists a unique internal equilibrium $P^{*}=\left(I^{*}, N^{*}, I^{*}, N^{*}\right)$ where

$$
R^{*}=\frac{\left(\beta_{1}+\beta_{2}+\frac{a r}{K}\right) \frac{r}{\frac{r}{K}(1+\alpha)}}{b+\gamma+\delta}, \quad I^{*}=\frac{r}{\delta}-\frac{r}{\delta K}(1+\alpha) N^{*}
$$

and $N^{*}$ is the larger solution of the equation

$$
\left(\beta_{1}+\beta_{2}\right) \frac{r}{\delta K}(1+\alpha) x^{2}+\left[\left(\beta_{1}+\beta_{2}\right)\left(1-\frac{r}{\delta}\right)-\frac{(1+\alpha-a) r}{K}\right] x-(d+\gamma+\delta)=0
$$


For this equilibrium, the Jacobian matrix is

$$
A^{*}=\left[\begin{array}{llll}
a_{1} & b_{1} & c_{1} & d_{1} \\
a_{2} & b_{2} & 0 & d_{2} \\
c_{1} & d_{1} & a_{1} & b_{1} \\
0 & d_{2} & a_{2} & b_{2}
\end{array}\right]
$$

where

$$
\begin{aligned}
& a_{1}=-\beta_{1} I^{*} N^{*}-\beta_{2} N^{*}+\delta I^{*}, \quad b_{1}=\beta_{1} I^{*}\left(1-I^{*}\right)+\frac{a r}{K} I^{*}, \quad c_{1}=\beta_{2}\left(1-I^{*}\right) N^{*}, \\
& d_{1}=\beta_{2}\left(1-I^{*}\right) I^{*}, \quad a_{2}=-\delta N^{*}, \quad b_{2}=-\frac{r}{K} N^{*}, \quad d_{2}=-\frac{r \alpha}{K} N^{*} .
\end{aligned}
$$

Through long computations, one finds that its characteristic equation is

$$
A_{0}+A_{1} \lambda+A_{2} \lambda^{2}+A_{3} \lambda^{3}+\lambda^{4}=0
$$

where

$$
\begin{aligned}
& A_{0}=\left(a_{1} b_{2}-a_{2} b_{1}\right)^{2}-\left(a_{2} d_{1}-a_{1} d_{2}\right)^{2}+2 a_{2} b_{2} c_{1} d_{1}-2 a_{2} b_{1} c_{1} d_{2}-b_{2}^{2} c_{1}^{2}+c_{1}^{2} d_{2}^{2}, \\
& A_{1}=-2\left(a_{1}+b_{2}\right)\left(a_{1} b_{2}-a_{2} b_{1}\right)-2 d_{2}\left(a_{2} d_{1}-a_{1} d_{2}\right)-2 a_{2} c_{1} d_{1}+2 b_{2} c_{1}^{2}, \\
& A_{2}=\left(a_{1}+b_{2}\right)^{2}-d_{2}^{2}-c_{1}^{2}+2\left(a_{1} b_{2}-a_{2} b_{1}\right), \quad A_{3}=-2\left(a_{1}+b_{2}\right),
\end{aligned}
$$

and its Hurwitz determinants are

$$
H_{1}=A_{3}, \quad H_{2}=A_{2} A_{3}-A_{1}, \quad H_{3}=A_{1} A_{2} A_{3}-A_{0} A_{3}^{2}-A_{1}^{2}, \quad H_{4}=A_{0} H_{3} .
$$

Clearly $A_{0}, A_{1}, A_{2}, A_{3}, H_{1}, H_{2}, H_{3}$ and $H_{4}$ are the functions of all the parameters of the system. In the following, we choose $\beta_{2}$ as the bifurcation parameter, and fix the other parameters. Because of the complexity of the characteristic equation, we present only numerical results, shown in Fig. 1, where $\beta_{2}$ varies from 0.14 to 0.18 . 

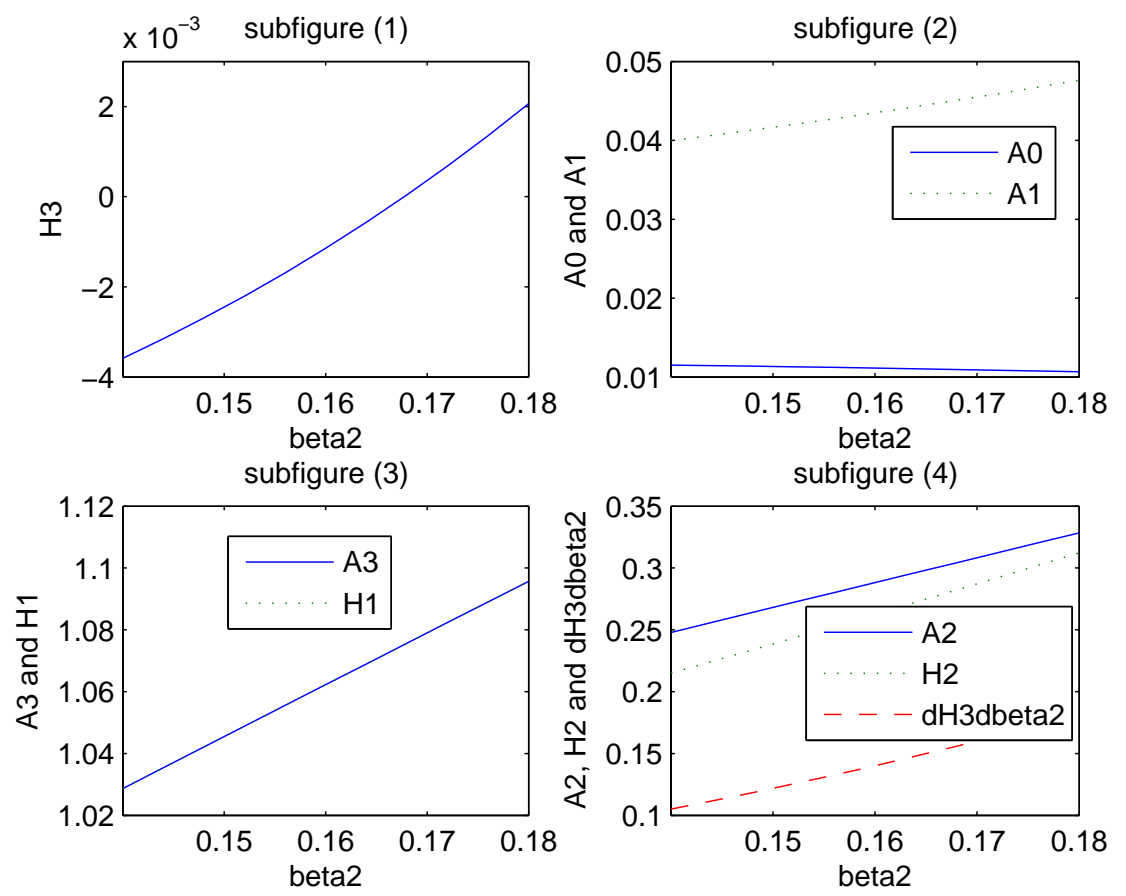

Fig. 1. the curves of $A_{0}, A_{1}, A_{2}, A_{3}, H_{1}, H_{2}, H_{3}$ and $\frac{d H_{3}}{d \beta_{2}}$ as functions of $\beta_{2}$. Parameter values are $r=1, b=2, a=0.5, K=2, d=1, \alpha=2, \delta=1, \gamma=1$ and $\beta_{1}=6$.

From Fig. 1, we see that, as $\beta_{2}$ changes from 0.14 to 0.18 , the values of $A_{0}, A_{1}, A_{2}, A_{3}, H_{1}, H_{2}, \frac{d H_{3}}{d \beta_{2}}$ always remain positive, but the value of $H_{3}$ changes from negative to positive. Hence, there exists some $\tilde{\beta}_{2} \in[0.14,0.18]$ such that $H_{3}\left(\tilde{\beta}_{2}\right)=0$ and $A_{0}, A_{1}, A_{2}, A_{3}, H_{1}, H_{2}, \frac{d H_{3}}{d \beta_{2}}$ are all positive. The conditions of Lemma 5.1 are satisfied and Hopf bifurcation occurs. So, the internal equilibrium $P^{*}$ bifurcates into a "small amplitude" periodic solution as $\beta_{2}$ passes through $\tilde{\beta}_{2}$.

Precisely, we find that, when $\beta_{2}=0.1677$ (in fact, this is just the value of $\tilde{\beta}_{2}$ in Fig. 1), Hopf bifurcation occurs at the internal equilibrium $P^{*}=$ $(0.038509,0.640994,0.038509,0.640994)$ and the corresponding Lyapunov coefficient $l=-23.93361$ is negative, which means that the periodic orbits are born stable (namely, stable limit cycles). In other words, as $\beta_{2}$ decreases 
through $\tilde{\beta}_{2}=0.1677$, the stability of the internal equilibrium changes from stable to unstable and a stable limit cycle arises. In Fig. 2, we show the stable limit cycle for $\beta_{2}=0.1$ (plotting, for simplicity, only the coordinates $I_{1}$ and $N_{1}$ of the limit cycle).

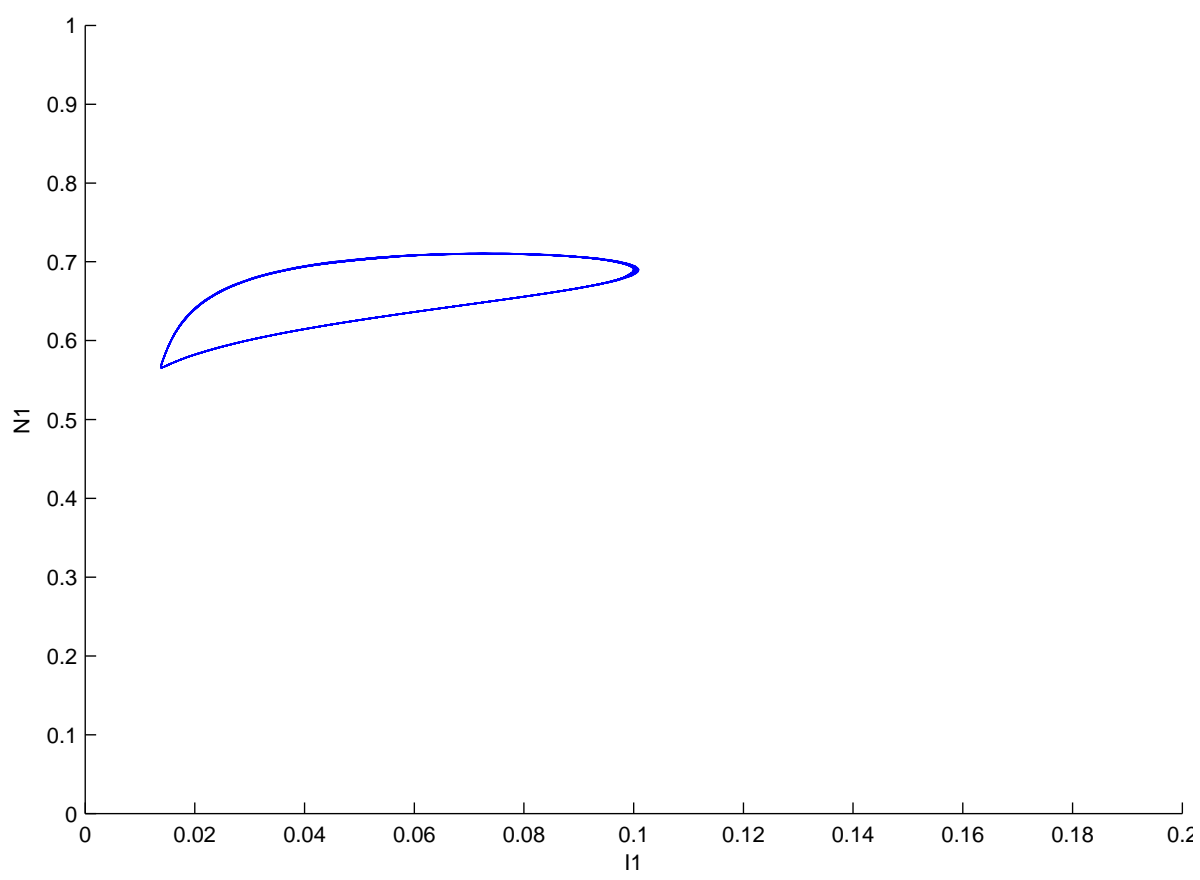

Fig. 2. The stable limit cycle for $\beta_{2}=0.1$

Let us moreover consider the stabilities of the boundary equilibria in this case. By numerical computations, we find that, for $\beta_{2}$ near $\tilde{\beta}_{2}=0.1677$, the equilibria $E_{0}, E_{1}, E_{2}, E_{12}$ are always unstable, but $B_{1}$ and $B_{2}$ are always stable. So we have the results as follows: (1) When $\beta_{2}>\tilde{\beta}_{2}=0.1677$, there exist three stable equilibria $B_{1}, B_{2}$ and $P^{*} ;(2)$ When $\beta_{2}<\tilde{\beta}_{2}=0.1677$, there exist two stable equilibria $B_{1}, B_{2}$ and a stable limit cycle.

Note that, with these parameter values, the pure competition model has both boundary equilibria $E_{1}$ and $E_{2}$ stable, and an internal unstable equilibrium $E_{12}$; i.e., the competition model is "bi-stable". Adding the infection, the sys- 
tem moves to a "tri-stable" situation: depending on the initial conditions, the solutions may settle to a state with either species alone with the endemic infection, or to a state with both species present and the infection either at an equilibrium, or, depending on the value of $\beta_{2}$, fluctuating in a limit cycle.

One can look at how the Hopf bifurcation depends on the other parameters. For instance in Fig. 3, we let $\beta_{2}$ and the inter-specific competition coefficient $\alpha$ vary; the points on the curve shown in that figure are all Hopf bifurcation points.

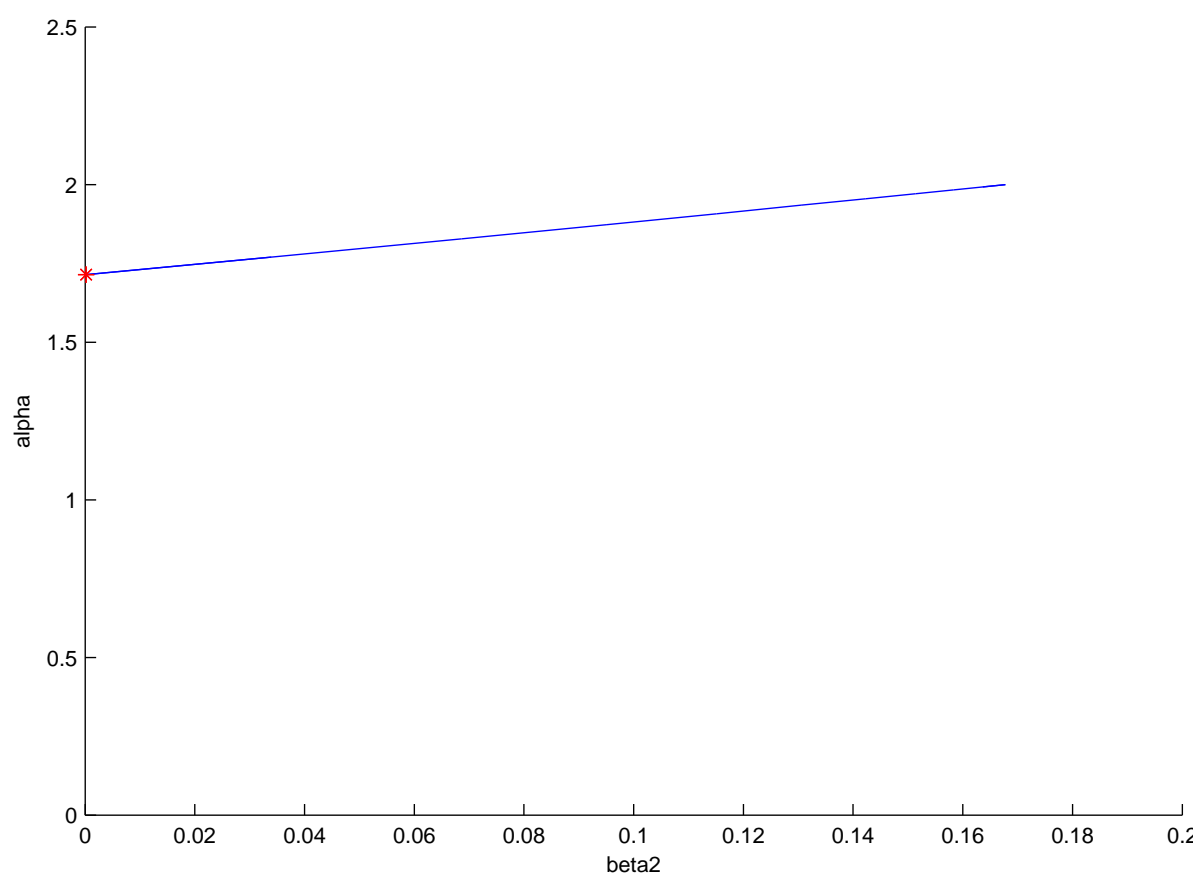

Fig. 3. the continuation curve of Hopf bifurcation points

This means that, for each point $\left(\tilde{\beta}_{2}, \tilde{\alpha}\right)$ on the curve, and $\alpha=\tilde{\alpha}$ is fixed, as $\beta_{2}$ decreases through $\tilde{\beta}_{2}$, the stability of the internal equilibrium $P^{*}$ changes from stable to unstable and a stable limit cycle occurs; similarly, if $\beta_{2}=\tilde{\beta}_{2}$ is fixed and $\alpha$ increases through $\tilde{\alpha}$. 
One can also check that, for values of $\left(\tilde{\beta}_{2}, \tilde{\alpha}\right)$ on the curve, the boundary equilibria $E_{0}, E_{1}, E_{2}, E_{12}$ are always unstable, while the equilibria $B_{1}$ and $B_{2}$ can be stable or unstable. This brings us important information, in agreement with [2]: (1) when all the boundary equilibria are unstable, the internal equilibrium may be stable or unstable; (2) when there exists a stable boundary equilibrium, the internal equilibrium may be stable or unstable; (3) the system may have three stable attractors and one of which is a limit cycle.

Finally, we present the bifurcation diagram in a special asymmetric case, in which all parameters

$$
\begin{gathered}
r_{1}=r_{2}=r, b_{1}=b_{2}=b, d_{1}=d_{2}=d, a_{1}=a_{2}=a, K_{1}=K_{2}=K, \\
\gamma_{1}=\gamma_{2}=\gamma, \delta_{1}=\delta_{2}=\delta, \beta_{11}=\beta_{22}=\beta_{1}, \beta_{12}=\beta_{21}=\beta_{2}
\end{gathered}
$$

are the same, except for the competition coefficients $\alpha_{12}$ and $\alpha_{21}$ that may be different.

We choose $\alpha_{21}$ as the bifurcation parameter, and fix the other parameters. With the help of MatCont numerical software [24], we obtain Fig. 4.

Fig. 4 shows (for simplicity, only the coordinate $N_{2}$ ) the bifurcation diagram of the internal equilibria and of the boundary equilibrium $B_{1}$ when $\alpha_{21}$ varies between 1.6 and 2.4. The stability is shown with the letters 's' (=stable) or ' $\mathrm{u}$ ' (=unstable), and the bifurcation points are shown as 'H' (Hopf bifurcation point), 'LP' (saddle-node bifurcation point) and 'BP' (branching point, or transcritical bifurcation point). From Fig. 4, one sees that: (1) when $\alpha_{21}<$ 1.7932, there is no internal equilibria; (2) when $1.7932<\alpha_{21}<1.8946$, there exist two internal equilibria, one is stable, the other is unstable; (3) when $1.8946<\alpha_{21}<1.9512$, there exist three internal equilibria, one is stable, 


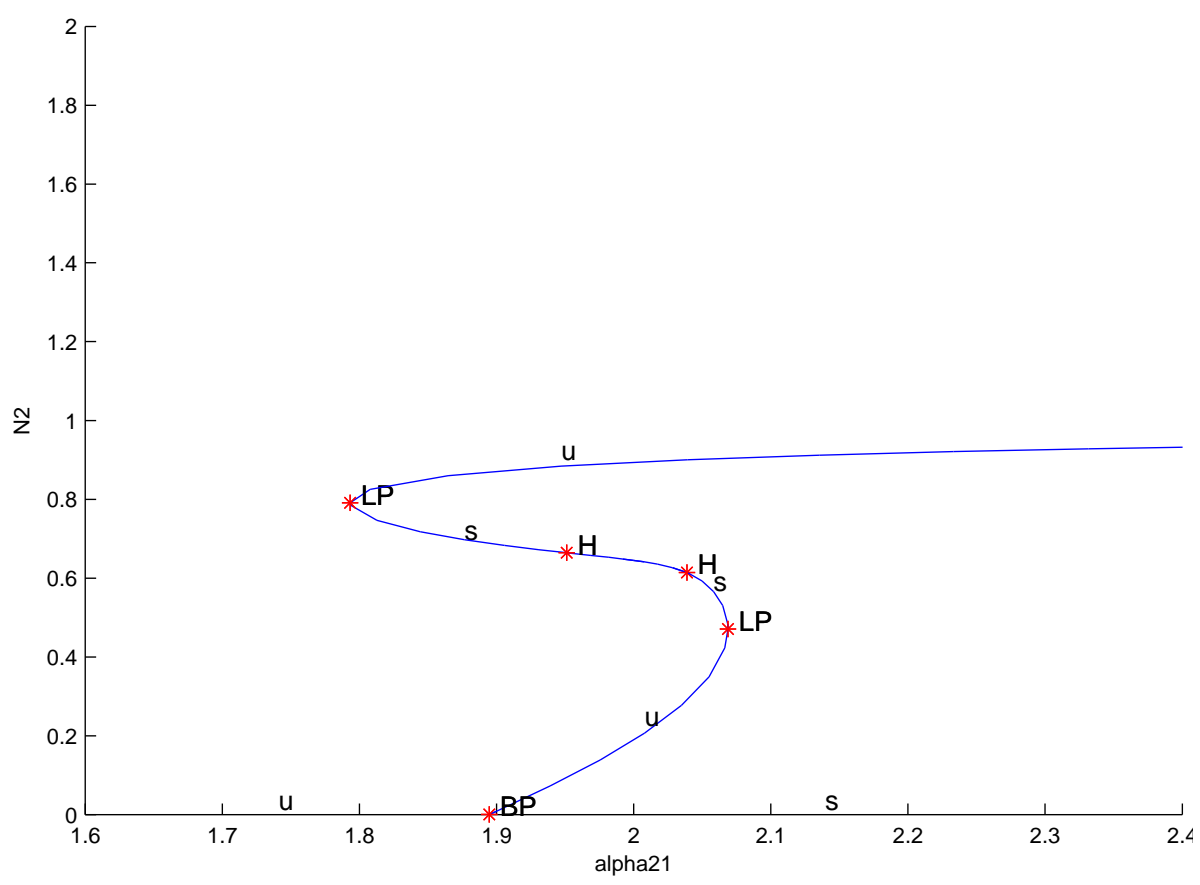

Fig. 4. the continuation curve of the internal equilibria. Parameter values are $r=1$, $b=2, a=0.5, K=2, d=1, \alpha_{12}=2, \delta=1, \gamma=1, \beta_{1}=6$ and $\beta_{2}=0.1$.

the other two are unstable; (4) when $1.9512<\alpha_{21}<2.0388$, there exist a stable limit cycle and three internal equilibria which are all unstable; (5) when $2.0388<\alpha_{21}<2.0688$, there exist three internal equilibria, one is stable, the other two are unstable; (6) when $\alpha_{21}>2.0688$, there exists an internal equilibrium which is unstable. In cases (1) and (2), the equilibrium $B_{1}$ is unstable, while it is stable in the other cases. Finally, it is possible to check that, independently of the value of $\alpha_{21}$, the equilibrium $B_{2}$ is always stable.

We have obtained the important information: (1) the system may have between zero and three internal equilibria; (2) with two stable boundary equilibrium, the system may have three internal equilibria, one of which may be stable. It is also possible that the system has three stable attractors, one of 
which is a limit cycle.

\section{Discussion}

In this paper, we considered an SIS epidemic model of two competitive species and obtained some results that show the biological significance and complicated behaviors of the model.

In general, one species does not exist alone in the natural world. It always interacts with other species for food, space and so on. Therefore, it is valuable to consider the effect of interacting species when we study the dynamical behaviors of epidemiological models. From this an interesting question occurs: how do infection and ecological interactions among species affect each other? In this competition model we found, by a careful analysis, important interactions between infection and competition as follows: (1) a species that would get extinct without the infection, may persist in presence of the infection; (2) a species that would coexist with its competitor without the infection, but is driven to extinction by the infection; (3) an infection that would die out in either species without the inter-infection of disease, may persist in both species in presence of this factor.

By mathematical analysis and numerical investigation, Holt and Pickering [7], Begon and Bowers $[1,8]$ conjectured that their models have the classic endemic model behavior with a unique attracting equilibrium, below and above the threshold. But Greenman and Hudson [2] did extensive analysis of these models and found counterexamples to this conjecture, showing that the two host epidemic models can have complicated behaviors. 
In our model, using Hopf bifurcation theory and numerical simulations, we have extended the analysis by Greenman and Hudson, revealing the following complicated behaviors: (1) when all the boundary equilibria are unstable, the internal equilibrium may be stable or unstable; (2) when there exists a stable boundary equilibrium, the internal equilibrium may exist or not, and be stable or unstable; (3) the system may have three stable attractors and one of which may be a limit cycle; (4) the system may have zero up to three internal equilibria; (5) when there exists a stable boundary equilibrium, the system may have three internal equilibria: sometimes all of them are unstable, but sometimes one of them is stable. Probably, several other interesting behaviors may be ascertained through a more extensive numerical investigation.

We have also analysed the system from the point of view of persistence. A much clearer picture emerges from this, and it is almost always possible to decide whether the system is persistent or not on the basis of a few quantities, related to the stability of the boundary equilibria (Table 4). A few cases remain partially undecided from this analysis. From Table 2 we find that host population $i(i=1,2)$ is weakly uniform persistent when $R_{j}>1, \pi_{i}>$ $0, \alpha_{i j} K_{j}>K_{i}, \alpha_{j i} K_{i}>K_{j}(i \neq j)$ and $R_{i}<1$ or $R_{i}>1, R_{12}<1$. For this case, we can not prove the strong uniform persistence; but we conjecture that there may exist some oscillating trajectory which destroys the strong uniform persistence.

Finally, we compare our model with the model by Saenz and Hethcote [3] who use the frequency-dependent incidence. In their model, there exists an extinction equilibrium $E^{*}=\left(I_{1}^{c}, 0, I_{2}^{c}, 0\right)$ stable under some conditions; this means that the infectious disease may drive both species to extinction. But in our model, there does not exist such an equilibrium, thus total extinction 
can not occur. It is in fact known that an infection may cause population extinction only with the frequency-dependent incidence, and not with the standard bilinear form, or other laws increasing with population density [25].

Another difference is that their model never has periodic solutions, nor multiple attractors, contrary to what we found in model (2.2). Hence, the different forms of the incidence can lead to rather different dynamical behaviors of the models.

At last, in their model, a key result is that the disease must either die out or remain endemic synchronously in both species. Which still occurs in our model, since the equilibrium value $I_{1}$ is zero if and only if $I_{2}$ is also zero. So, the occurrence of this behavior is independent of the form of the incidence.

\section{References}

[1] M. Begon, R. G. Bowers, N. Kadianakis and D. E. Hodgkinson, Disease and community structure: The importance of host self-regulation in a host-hostpathogen model, Am. Nat. 139 (1992), 1131-1150.

[2] J. V. Greenman and P. J. Hudson, Infected coexistence instability with and without density-dependent regulation, J. Theor. Biol. 185 (1997), 345-356.

[3] R. A. Saenz and H. W. Hethcote, Competing species models with an infectious disease, Math. Biosci. Eng. 3 (2006), 219-235.

[4] M. Begon, S. M. Hazel, D. Baxby, K. Bown, R. Cavanagh, J. Chantrey, T. Jones and M. Bennett, Transmission dynamics of a zoonotic pathogen within and between wildlife host species, Proc. R. Soc. Lond. B 266 (1999), 1939-1945.

[5] D. M. Tompkins, A. R. White and M. Boots, Ecological replacement of native 
red squirrels by invasive greys driven by disease, Ecology Letters 6 (2003), 189196.

[6] M. Begon, S. M. Hazel, S. Telfer, K. Bown, D. Carslake, R. Cavanagh, J. Chantrey, T. Jones and M. Bennett, Rodents, cowpox virus and islands: Densities, numbers and thresholds, J. Animal Ecology 2 (2003), 343-355.

[7] R. D. Holt and J. Pickering, Infectious disease and species coexistence: A model of Lotka-Volterra form, Am. Nat. 126 (1985), 196-211.

[8] M. Begon and R. G. Bowers, Beyond host-pathogen dynamics, in "Ecology of disease in natural populations" (Edited by B. T. Grenfell and A. P. Dobson), Cambridge University Press, Cambridge (1995), 479-509.

[9] H. W. Hethcote, W. Wang and Y. Li, Species coexistence and periodicity in host-host-pathogen models, J. Math. Biol. 51 (2005), 629-660.

[10] R. M. Anderson and R. M. May, The invasion, persistence, and spread of infectious diseases within animal and plant communites, Phil. Trans. R. Soc. Lond. B 314 (1986), 533-570.

[11] R. G. Bowers and J. Turner, Community structure and the interplay between interspecific infection and competition, J. Theor. Biol. 187 (1997), 95-109.

[12] E. Venturino, The effects of diseases on competing species, Math. Biosci. 174 (2001), 111-131.

[13] L. Han, Z. Ma and T. Shi, An SIRS epidemic model of two competitive species, Mathl. Comput. Modelling 37 (2003), 87-108.

[14] H. R. Thieme, Persistence under relaxed point-dissipativity (with application to an endemic model), SIAM J. Math. Anal. 24 (1993), 407-435.

[15] L. Q. Gao and H. W. Hethcote, Disease transmission models with densitydependent demographics, J. Math. Biol. 30 (1992), 717-731. 
[16] J. Zhou and H. W. Hethcote, Population size dependent incidence in models for diseases without immunity, J. Math. Biol. 32 (1994), 809-834.

[17] O. Diekmann, J. A. P. Heesterbeek and J. A. J. Metz, On the definition and the computation of the basic reproduction ratio $R_{0}$ in models for infectious diseases in heterogeneous populations, J. Math. Biol. 28 (1990), 365-382.

[18] A. Pugliese, Population models for disease with no recovery, J. Math. Biol. 28 (1990), 65-82.

[19] O. Diekmann and J. A. P. Heesterbeek, Mathematical epidemiology of infectious diseases, Wiley, New York, 2000.

[20] R. Rosà and A. Pugliese, Effects of tick population dynamics and host densities on the persistence of tick-borne infections, Math. Biosci., 208 (2007), 216-240.

[21] P. Magal and X. Zhao, Global attractors and steady states for uniformly persistent dynamical systems, SIAM J. Math. Anal. 37 (2005), 251-275.

[22] J. Hofbauer and K. Sigmund, The theory of evolution and dynamical systems, Cambridge University Press, Cambridge, 1988.

[23] J. Shen and Z. Jing, A new detecting method for conditions of existence of Hopf bifurcation, Acta Math. Appl. Sinica 11 (1995), 79-93.

[24] MATCONT, http://www.matcont.ugent.be

[25] H. R. Thieme, Epidemic and demographic interaction in the spread of potentially fatal diseases in growing population, Math. Biosci. 111 (1992), 99130. 